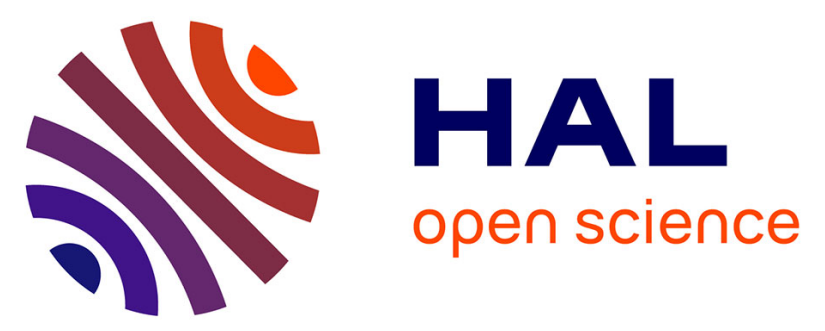

\title{
Effective predictions of heterogeneous flexoelectric multilayered composite with generalized periodicity
}

D. Guinovart-Sanjuán, K. Vajravelu, R. Rodríguez-Ramos, R. Guinovart-Díaz, J. Bravo-Castillero, Frédéric Lebon, F.J. Sabina, J. Merodio

\section{- To cite this version:}

D. Guinovart-Sanjuán, K. Vajravelu, R. Rodríguez-Ramos, R. Guinovart-Díaz, J. Bravo-Castillero, et al.. Effective predictions of heterogeneous flexoelectric multilayered composite with generalized periodicity. International Journal of Mechanical Sciences, 2020, 181, pp.105755. 10.1016/j.ijmecsci.2020.105755 . hal-02909276

\section{HAL Id: hal-02909276 \\ https://hal.science/hal-02909276}

Submitted on 3 Sep 2020

HAL is a multi-disciplinary open access archive for the deposit and dissemination of scientific research documents, whether they are published or not. The documents may come from teaching and research institutions in France or abroad, or from public or private research centers.
L'archive ouverte pluridisciplinaire HAL, est destinée au dépôt et à la diffusion de documents scientifiques de niveau recherche, publiés ou non, émanant des établissements d'enseignement et de recherche français ou étrangers, des laboratoires publics ou privés.

\section{(c)(1)}

Distributed under a Creative Commons Attribution| 4.0 International License 


\title{
Effective predictions of heterogeneous flexoelectric multilayered composite with generalized periodicity
}

\author{
D. Guinovart-Sanjuán ${ }^{\mathrm{a}}$, K. Vajravelu ${ }^{\mathrm{a}}$, R. Rodríguez-Ramos ${ }^{\mathrm{b}, *}$, R. Guinovart-Díaz ${ }^{\mathrm{b}}$, \\ J. Bravo-Castillero ${ }^{d}$, F. Lebon ${ }^{\text {c }}$, F.J. Sabina ${ }^{\mathrm{d}}$, J. Merodio ${ }^{\mathrm{e}}$

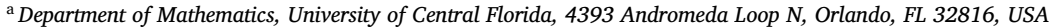 \\ ${ }^{\mathrm{b}}$ Departamento de Matemática, Universidad de La Habana, San Lazaro y L, La Habana, 10400, Cuba \\ ${ }^{\text {c } A i x-M a r s e i l l e ~ U n i v e r s i t y, ~ C N R S, ~ C e n t r a l e ~ M a r s e i l l e, ~ L M A, ~} 4$ Impasse Nikola Tesla, CS 40006, Marseille Cedex 13 13453, France \\ ${ }^{\mathrm{d}}$ Instituto de Investigaciones en Matemááticas Aplicadas y en Sistemas, Universidad Nacional Autónoma de México, Delegación Álvaro Obregón, Apartado Postal \\ 20-726, CDMX 01000, México \\ e Departamento de Mecánica de los Medios Continuos y T. Estructuras, E.T.S. de Caminos, Canales y Puertos, Universidad Politécnica de Madrid, C.P. 28040 Madrid, \\ España
}

In this work, the general mathematical statements for flexoelectric heterogeneous equilibrium boundary value problems are reported. A methodology to find the local problems and the effective properties of flexoelectric composites with generalized periodicity is presented, using the two-scales asymptotic homogenization method. The statement of the homogenized boundary values problem is given. A procedure to solve the local problems of stratified multilayered composites with complex geometry and perfect contact at the interface is proposed. Consequently, the analytical expressions of the effective coefficients are obtained. The piezoelectric limit case for rectangular bi-laminated composites is validated. Finally, numerical analysis to illustrate the behavior of the effective properties for rectangular and wavy flexoelectric bi-layered structures are shown.

\section{Introduction}

In recent years, there has been aincreased interest in the study of the piezoelectric and flexoelectric properties of the materials. Many authors have highlighted the main difference between these two classes of phenomena [1]. Piezoelectricity and flexoelectricity are linear electromechanical coupling. While piezoelectric effect describes the generation of electric polarization under homogeneous deformation (uniform mechanical strain) [2], flexoelectric effect refers to the electric polarization under strain gradient $[3,4]$.

Piezoelectric composites are widely used in energy harvesting, acoustic sensors and the development of actuators and transducers able to operate at elevated temperatures $[5,6]$. Flexoelectricity has been observed in several systems such as isotropic elastomers, liquid crystal, and crystal plates [7]. The flexoelectric phenomenon in biological materials such as bones and bio-membranes $[8,9]$, has been studied by many authors, due to the applications in biomaterials engineering. For example, the flexoelectric behavior of the bones is essential for bone's self-repair and remodeling properties [10]. According to the authors, the flexoelectricity has a central role in the crack-healing process of the bones. On the other hand, the flexoelectric materials like $\mathrm{BaTiO}_{3}$ have been used to fabricate nanogenerators that harvest energy from light, mechanical vibration and heat more efficiently [11]. In [12], other applications of flexoelectricity are presented, like the use of flexoelectric materials in the fabrication of nano-sized devices for sensors and actuators.

Due to the multiple combinations of materials, the composite structures with elastic, piezoelectric or flexoelectric components are widely studied. This work presents a method to study flexoelectric composite with generalized periodicity, taking the equilibrium equations reported in [13] as the object of study. In [13], the authors deduced the equilibrium problem from the electric Gibbs energy density. The flexoelectric part of the equation is characterize by a fourth order tensor $\boldsymbol{\mu}$. It is mentioned that $\boldsymbol{\mu}$ tensor is a combination of the direct and inverse flexoelectric tensors, which is studied in [1]. Many authors have brought different approaches to solve or approximate the solutions of the equilibrium equations. One of the most common methods is to find the homogenized problem using the two scales asymptotic homogenization method (AHM). In [14-16], the authors offer a detailed description of the steps to find effective properties of elastic/piezoelectric rectangular composites applying AHM. On the other hand, in [17], a methodology to find the effective properties for the case of multilayered materials is presented. The concept of generalized periodicity used in [17] consid-

\footnotetext{
* Corresponding author.

E-mail address: reinaldo@matcom.uh.cu (R. Rodríguez-Ramos).
} 
ers that the properties of the materials are periodic with respect to a stratification function, $\boldsymbol{\rho}(\mathbf{x})$, that describes the geometry of the layers. The results described in [17] are extended in [18] to elastic curvilinear structures with perfect and imperfect contacts at the interface. Now, the present work applies the concept of structure with generalized periodicity to flexoelectric composites and describes a procedure to find the effective coefficients from the local problems that arise from AHM. Since the flexoelectric effect is considered, the results shown in [16,17] and [19] are particular cases of the present work.

It is important to highlight that the asymptotic homogenization method is rigorously justified [20]. The averaging method is efficient for investigating macroscopic and microscopic properties of periodic structures, see [21]. One of the main advantages of the described technique is the possibility of determining the effective properties of a large number of periodic composites. In the case of flexoelectric structures, the analytical expressions of local problems and the effective coefficients can be derived. The equivalent homogenized equilibrium problem with nonrapidly oscillating coefficients for a flexoelectric composite is obtained from the effective properties. In this manuscript, the analytical solutions of the local problems for flexoelectric materials with generalized periodicity are presented for the particular case of stratified composite, as a generalization of the procedure described in [17] which is for the case of elastic composites.

In Section 2, the general equations of the equilibrium problem for a flexoelectric composite with generalized periodicity are reported. Some remarks about the matrix representation of the constitutive relations and the flexoelectric tensor are given in Appendix A. In Section 3, the procedure of the two-scales asymptotic homogenization method is presented. The displacements and electrical fields are written in terms of the expansion. The consequences for different powers of the small parameter are considered. The mathematical statement of the homogenized flexoelectric equilibrium problem is given. Consequently, in Section 3.1 the general expressions of the local problems for a flexoelectric composite are yielded. The details are specified in Appendix B. The conditions to guarantee the existence and uniqueness of the solutions of the local problems are mentioned in Appendix C. An algorithm to obtain the effective properties is reported in Section 3.2. Finally, in Section 4 some numerical examples are illustrated. The procedure described in the previous section is validated with the results reported in [22], considering a piezocomposite as a particular case of a flexoelectric heterogeneous structure. On the other hand, two numerical cases of stratified flexoelectric composites are studied. The first example illustrates the effective properties of a bi-materials rectangular laminate composite where the constituents are flexoelectric materials with cubic symmetry. The second example deals with the computation of the macroscopic characteristics for a wavy periodic bi-laminated medium with the same material parameters, considering the dependence of the coefficients with respect to the stratification function. The solution of the local problems for stratified structures is introduced in the Appendix D.

\section{Equilibrium equation}

Let $\Omega \subset \mathbb{R}^{3}$ be a three-dimensional open connected bounded domain with an infinitely smooth boundary $\partial \Omega$. The equilibrium problem on a flexoelectric structure $\Omega$ is given by the equation

$\partial_{j} \sigma_{i j}+f_{i}=0, \quad$ in $\Omega$,

$\partial_{i} D_{i}=0, \quad$ in $\Omega$,

and boundary conditions

$u_{i}=u_{i}^{0}$, on $\partial \Omega_{u}, \quad t_{i}=t_{i}^{0}$, on $\partial \Omega_{t}$,

$\partial_{j} u_{i} n_{j}=s_{i}^{0}$, on $\partial \Omega_{s}, \quad \mu_{l i j k} E_{l} n_{i} n_{j}=r_{k}^{0}$, on $\partial \Omega_{r}$,

$\phi=\phi^{0}$, on $\partial \Omega_{\phi}, \quad D_{i} n_{i}=-\tau$, on $\partial \Omega_{\tau}$,

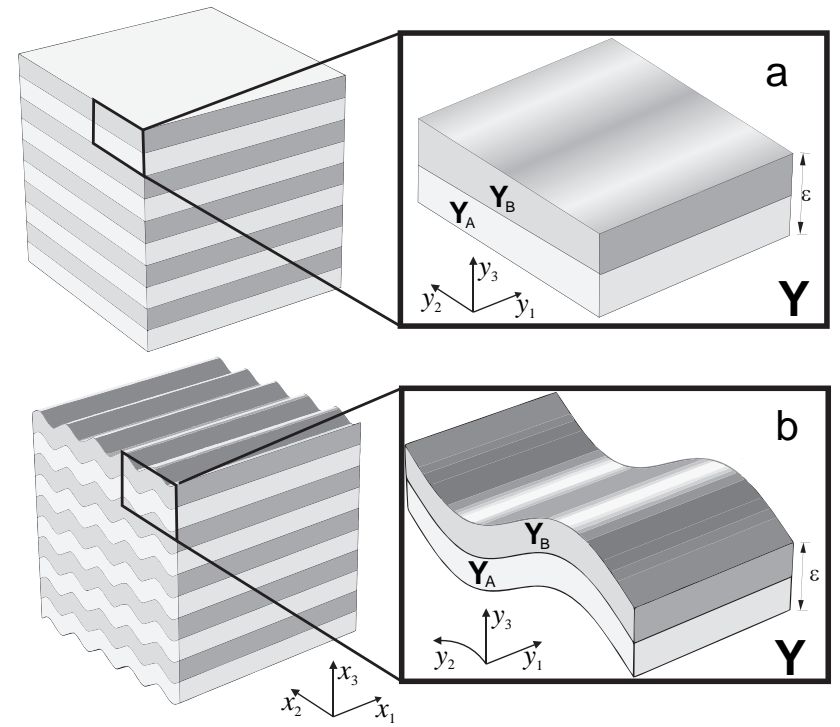

Fig. 1. Flexoelectric multilayered composite: a) rectangular geometry at the periodic cell and b) with complex geometrical shape microstructure.

where $\partial_{k}(\bullet)=\partial(\bullet) / \partial x_{k} ; \boldsymbol{\sigma}$ is the general stress tensor; $\mathbf{f}$ is the external forces vector; $\mathbf{D}$ is the electric displacement vector; $\mathbf{u}, \mathbf{u}^{0}$ are the displacement and prescribed displacement vectors, respectively; $\mathbf{n}$ is the normal vector to the corresponding surface; $\mathbf{E}$ is the electric field vector; $\mathbf{t}, \mathbf{t}^{0}$ are the traction and prescribed traction vectors, respectively; $\mathbf{s}^{0}$ is the prescribed normal derivative of the displacement vector and $\phi, \phi^{0}, \mathbf{r}^{0}, \tau$ are the electrical potential, the prescribed electrical potential, prescribed higher-order traction and surface charge, respectively; where $\partial \Omega \equiv \partial \Omega_{u} \cup \partial \Omega_{t} \equiv \partial \Omega_{s} \cup \partial \Omega_{r} \equiv \partial \Omega_{\phi} \cup \partial \Omega_{\tau}$ and $\emptyset \equiv \partial \Omega_{u} \cap \partial \Omega_{t} \equiv \partial \Omega_{s} \cap \partial \Omega_{r} \equiv \partial \Omega_{\phi} \cap \partial \Omega_{\tau}$.

The constitutive relationships between stress $\sigma$, electrical displacement $\mathbf{D}$, strain $\boldsymbol{\epsilon}$ and electrical field $\mathbf{E}$ have the following form written by components

$\sigma_{i j}=C_{i j k l} \epsilon_{k l}-e_{k i j} E_{k}+\mu_{l i j k} \partial_{k} E_{l}$,

$D_{i}=e_{i k l} \epsilon_{k l}+\mu_{i j k l} \partial_{l} \epsilon_{j k}+\kappa_{i j} E_{j}$,

where $\mathbf{C}, \mathbf{e}, \boldsymbol{\kappa}$ and $\boldsymbol{\mu}$ denote the stiffness, piezoelectric, permittivity and flexoelectric tensors, respectively. The matrix representation of the constitutive relations (6)-(7) and some explanations about the flexoelectric tensor are reported in Appendix A. For small deformations, the strain $\boldsymbol{\epsilon}$ and the electrical field $\mathbf{E}$ relate to the displacement tensor $\mathbf{u}$ and the electrical potential $\phi$ as

$\epsilon_{i j}=\frac{1}{2}\left(\partial_{j} u_{i}+\partial_{i} u_{j}\right), \quad E_{j}=-\partial_{j} \phi$,

The mathematical statement for a flexoelectric media is derived substituting (6)-(8) into (1)-(2) under boundary conditions (3)-(5).

The mechanical and electrical properties of a curvilinear flexoelectric periodic heterogeneous structure, $\mathbf{C}^{\varepsilon} \equiv \mathbf{C}^{\varepsilon}(\mathbf{x}, \mathbf{y}), \mathbf{e}^{\varepsilon} \equiv \mathbf{e}^{\varepsilon}(\mathbf{x}, \mathbf{y})$, $\boldsymbol{\kappa}^{\varepsilon} \equiv \boldsymbol{\kappa}^{\varepsilon}(\mathbf{x}, \mathbf{y})$, and $\boldsymbol{\mu}^{\varepsilon} \equiv \boldsymbol{\mu}^{\varepsilon}(\mathbf{x}, \mathbf{y})$ are regular functions with respect the variable $\mathbf{x} \in \mathbb{R}$ and periodic with respect the variable $\mathbf{y}=\boldsymbol{\rho}(\mathbf{x}) / \varepsilon \in \mathbf{Y}$, where $\boldsymbol{\rho}(\mathbf{x})$ is the surface that describes the geometry, $0<\varepsilon<<1$ is a very small parameter that characterizes the heterogeneity of the structure and $\mathbf{Y} \subset \mathbb{R}^{3}$ is called the periodic cell. As an example, a bimaterials multilayered flexoelectric composite with generalized periodicity is shown in Fig. 1b. The geometry of the periodic cell $\mathbf{Y}$ is described by the function $\boldsymbol{\rho}(\mathbf{x})$. The small parameter $\varepsilon$ characterizes the periodicity of the layers. The variable $\mathbf{y}=\boldsymbol{\rho}(\mathbf{x}) / \varepsilon$ has a wavy behavior due to the presence of $\boldsymbol{\rho}(\mathbf{x})$. In this particular case, the wavy is changing along $y_{2}$, the layers are transversal to the $y_{3}$ axis, while the properties are constant along the $y_{1}$ axis. 

by

The material properties satisfy the usual symmetry conditions given

$C_{i j k l}^{\varepsilon}=C_{j i k l}^{\varepsilon}=C_{i j l k}^{\varepsilon}=C_{k l i j}^{\varepsilon}, \quad e_{i j k}^{\varepsilon}=e_{i k j}^{\varepsilon}, \quad \kappa_{i j}^{\varepsilon}=\kappa_{j i}^{\varepsilon}$.

Substituting the formulas (6)-(8) into the Eqs. (1)-(5), based on the slow $\mathbf{x}$ variable and rapid $\mathbf{y}$ variable, the heterogeneous flexoelectric equilibrium problem for a composite with perfect contact at the interface, can be written, using the total derivative operator $\partial_{j}(\bullet)=\partial(\bullet) / \partial x_{j}+$ $\left(\varrho_{s, j} / \varepsilon\right) \partial(\bullet) / \partial y_{s}$ as

$$
\begin{aligned}
& \left(\frac{\varrho_{m, j}}{\varepsilon} C_{i j k l \mid m}^{\varepsilon}+C_{i j k l, j}^{\varepsilon}\right) u_{k, l}^{\varepsilon}+C_{i j k l}^{\varepsilon} u_{k, l j}^{\varepsilon}+\left(\frac{\varrho_{l, j}}{\varepsilon} e_{k i j \mid l}^{\varepsilon}+e_{k i j, j}^{\varepsilon}\right) \phi_{, k}^{\varepsilon} \\
& +e_{k i j}^{\varepsilon} \phi_{, k j}^{\varepsilon}-\left(\frac{\varrho_{m, j}}{\varepsilon} \mu_{l i j k \mid m}^{\varepsilon}+\mu_{l i j k, j}^{\varepsilon}\right) \phi_{, l k}^{\varepsilon}-\mu_{l i j k}^{\varepsilon} \phi_{, l k j}^{\varepsilon}+f_{i}=0, \\
& \left(\frac{\varrho_{s, i}}{\varepsilon} e_{i k l \mid s}^{\varepsilon}+e_{i k l, i}^{\varepsilon}\right) u_{k, l}^{\varepsilon}+e_{i k l}^{\varepsilon} u_{k, l i}^{\varepsilon}+\left(\frac{\varrho_{s, i}}{\varepsilon} \mu_{i j k l \mid s}^{\varepsilon}+\mu_{i j k l, i}^{\varepsilon}\right) u_{j, k l}^{\varepsilon} \\
& +\mu_{i j k l}^{\varepsilon} u_{j, k l i}^{\varepsilon}-\left(\frac{\varrho_{s, i}}{\varepsilon} \kappa_{i j \mid s}^{\varepsilon}+\kappa_{i j, i}^{\varepsilon}\right) \phi_{, j}^{\varepsilon}-\kappa_{i j}^{\varepsilon} \phi_{, j i}^{\varepsilon}=0,
\end{aligned}
$$

where $(\bullet)_{\mid s}=\partial(\bullet) / \partial y_{s},(\bullet)_{, s}=\partial(\bullet) / \partial x_{s}$, with boundary conditions

$u_{i}^{\varepsilon}=u_{i}^{0}$, on $\partial \Omega_{u}, \quad t_{i}^{\varepsilon}=t_{i}^{0}$, on $\partial \Omega_{t}$,

$u_{i, j}^{\varepsilon} n_{j}=s_{i}^{0}$, on $\partial \Omega_{s}, \quad n_{i} n_{j} \mu_{l i j k}^{\varepsilon} E_{l}^{\varepsilon}=r_{i}^{0}$, on $\partial \Omega_{r}$,

$\phi^{\varepsilon}=\phi^{0}$, on $\partial \Omega_{\phi}, \quad D_{i}^{\varepsilon} n_{i}=-\tau$, on $\partial \Omega_{\tau}$,

and continuity conditions at interface

$\left[\left[u_{i}^{\varepsilon}\right]\right]=0,\left[\left[\phi^{\varepsilon}\right]\right]=0,\left[\left[\sigma^{\varepsilon}{ }_{i j} n_{j}\right]\right]=0,\left[\left[D_{j}^{\varepsilon} n_{j}\right]\right]=0$, on $\Gamma$,

where $\Gamma$ is the interface surface between two different components. The Eq. (15) implies that the stress tensor $\sigma$, the displacement vector $\mathbf{u}$, the electrical displacement vector $\mathbf{D}$ and the electric potential $\phi$ are considered continuous functions at the interface (perfect contact condition).

The problem (10)-(14) for the particular case when $\varrho_{i, j}=\delta_{i j}$, is reported in [13]. On the other hand, the equilibrium problem (10)-(14) is a generalization of Eq. (6)-(7) obtained in [23] for the case of an elastic structure.

\section{Asymptotic homogenization method}

The basic idea is to expand the fields in term of the aforementioned small parameter. Therefore, the asymptotic expansions for the functions $\sigma_{i j}^{\varepsilon}, D_{i}^{\varepsilon}, u_{k}^{\varepsilon}$ and $\phi^{\varepsilon}$ are given by

$u_{k}^{\varepsilon}(\mathbf{x}, \mathbf{y})=u_{k}^{(0)}(\mathbf{x})+\varepsilon u_{k}^{(1)}(\mathbf{x}, \mathbf{y})+\varepsilon^{2} u_{k}^{(2)}(\mathbf{x}, \mathbf{y})+\ldots$,

$\phi^{\varepsilon}(\mathbf{x}, \mathbf{y})=\phi^{(0)}(\mathbf{x})+\varepsilon \phi^{(1)}(\mathbf{x}, \mathbf{y})+\varepsilon^{2} \phi^{(2)}(\mathbf{x}, \mathbf{y})+\ldots$,

$\sigma_{i j}^{\varepsilon}(\mathbf{x}, \mathbf{y})=\sigma_{i j}^{(0)}(\mathbf{x}, \mathbf{y})+\varepsilon \sigma_{i j}^{(1)}(\mathbf{x}, \mathbf{y})+\varepsilon^{2} \sigma_{i j}^{(2)}(\mathbf{x}, \mathbf{y})+\ldots$,

$D_{i}^{\varepsilon}(\mathbf{x}, \mathbf{y})=D_{i}^{(0)}(\mathbf{x}, \mathbf{y})+\varepsilon D_{i}^{(1)}(\mathbf{x}, \mathbf{y})+\varepsilon^{2} D_{i}^{(2)}(\mathbf{x}, \mathbf{y})+\ldots$

The fact that $u_{k}^{(0)}$ and $\phi^{(0)}$ depend only on the macrovariable $\mathbf{x}$ can be proved by the same procedure presented in Section 5 of the Introduction of [21], see Eq. (3)-(5).

From now on, for simplicity the superscript " $\varepsilon$ " on the magnitudes is omitted. The asymptotic expansions (16)-(17) are substituted into constitutive relationships (6)-(8), see Appendix B, Eqs. (B.1)-(B.2). In order to avoid singularities when $\varepsilon \rightarrow 0$, the coefficients for $\varepsilon^{-1}$ are equated to zero

$\mu_{l i j k} \varrho_{r, l} \varrho_{s, k} \phi_{\mid r s}^{(1)}=0, \quad \mu_{i k j l} \varrho_{r, l} \varrho_{s, j} u_{k \mid r s}^{(1)}=0$.
Consequently, the following recurrent formulas for $\sigma_{i j}^{(n)}$ and $D_{i}^{(n)}$ are derived from (B.3)-(B.4), for $\varepsilon^{n}, n \geq 0$

$$
\begin{aligned}
\sigma_{i j}^{(n)}= & C_{i j k l} u_{k, l}^{(n)}+C_{i j k l} \varrho_{r, l} u_{k \mid r}^{(n+1)}+e_{k i j} \phi_{, j}^{(n)}+e_{k i j} \varrho_{r, k} \phi_{\mid r}^{(n+1)} \\
& -\mu_{l i j k} \phi_{, l k}^{(n)}-\left(\mu_{k i j l}+\mu_{l i j k}\right) \varrho_{r, l} \phi_{\mid r, k}^{(n+1)}-\mu_{l i j k} \varrho_{r, l} \varrho_{s, k} \phi_{\mid r s}^{(n+2)}, \\
D_{i}^{(n)}= & e_{i k l} u_{k, l}^{(n)}+e_{i k l} \varrho_{r, l} l_{k \mid r}^{(n+1)}-\kappa_{i j} \phi_{, j}^{(n)}-\kappa_{i j} \varrho_{s, j} \phi_{\mid s}^{(n+1)} \\
& +\mu_{i k j l} u_{k, l j}^{(n)}+\left(\mu_{i k l j}+\mu_{i k j l}\right) \varrho_{r, l} u_{k, j \mid r}^{(n+1)}+\mu_{i k j l} \varrho_{r, l} \varrho_{s, j} u_{k \mid r s}^{(n+2)} .
\end{aligned}
$$

Equating to zero the contributions of the terms $\varepsilon^{-1}$ and $\varepsilon^{0}$ derived from (B.5)-(B.6), the following equations are obtained

$\left(\varepsilon^{-1}\right): \varrho_{k, j} \sigma_{i j \mid k}^{(0)}=0, \quad \varrho_{k, i} D_{i \mid k}^{(0)}=0$,

$\left(\varepsilon^{0}\right): \sigma_{i j, j}^{(0)}+\varrho_{k, j} \sigma_{i j \mid k}^{(1)}+f_{i}=0, \quad D_{i, i}^{(0)}+\varrho_{k, i} D_{i \mid k}^{(1)}=0$.

It is known that for the AHM, the first order approximation gives a good approach for the solution of the heterogeneous equilibrium problem (1)(2) and it has an accuracy of order $\varepsilon^{1 / 2}$, see Theorem 6.3 in [20] and Chapter 6 in [21]. Hence, the higher order of $\varepsilon$ are neglected. The homogenized equations of a flexoelectric medium can be obtained applying the average operator $\langle\bullet\rangle=1 /|\mathbf{Y}| \int_{\mathbf{Y}}(\bullet) d \mathbf{y}$ to the Eq. (24) and considering the periodicity of $\sigma_{i j}^{(1)}$ and $D_{i}^{(1)}$ with respect the variable $\mathbf{y}$. Thus, the homogenized problem takes the form,

$\hat{\sigma}_{i j, j}+f_{i}=0$,

$\hat{D}_{i, i}=0$,

where $\hat{\sigma}_{i j}=\left\langle\sigma_{i j}^{(0)}\right\rangle$ and $\hat{D}_{i}=\left\langle D_{i}^{(0)}\right\rangle$. It is known that the solution of the heterogeneous equilibrium problem (10)-(14) approaches to the solution of the homogeneous problem as $\varepsilon \rightarrow 0$, using the two-scales asymptotic homogenization method.

To find an expression for $\sigma_{i j}^{(0)}$ and $D_{i}^{(0)}$, the system of partial differential Eq. (23) must be solved. Therefore, the expressions of $\sigma_{i j}^{(0)}$ and $D_{i}^{(0)}$ derived from (21)-(22) for $n=0$, are substituted into (23),

$$
\begin{aligned}
& \varrho_{t, j}\left(C_{i j k l} u_{k, l}^{(0)}+C_{i j k l} \varrho_{r, l} u_{k \mid r}^{(1)}+e_{j i k} \phi_{, k}^{(0)}+e_{j i k} \varrho_{r, k} \phi_{\mid r}^{(1)}\right. \\
& \left.-\mu_{l i j k} \phi_{, l k}^{(0)}-\left(\mu_{k i j l}+\mu_{l i j k}\right) \varrho_{r, l} \phi_{\mid r, k}^{(1)}-\mu_{l i j k} \varrho_{r, l} \varrho_{s, k} \phi_{\mid r s}^{(2)}\right)_{\mid t}=0, \\
& \varrho_{t, j}\left(e_{i k l} u_{k, l}^{(0)}+e_{i k l} \varrho_{r, l} u_{k \mid r}^{(1)}-\kappa_{i j} \phi_{, j}^{(0)}-\kappa_{i j} \varrho_{s, j} \phi_{\mid s}^{(1)}\right. \\
& \left.+\mu_{i k j l} u_{k, l j}^{(0)}+\left(\mu_{i k l j}+\mu_{i k j l}\right) \varrho_{r, l} u_{k, j \mid r}^{(1)}+\mu_{i k j l} \varrho_{r, l} \varrho_{s, j} u_{k \mid r s}^{(2)}\right)_{\mid t}=0 .
\end{aligned}
$$

Due to the linearity of the system (27)-(28) and assuming the regularity of the components and the smoothness in variation of the coefficients, the following decomposition of the functions $u_{k}^{(n)}$ and $\phi^{(n)}, n \geq 1$, is considered [21],

$u_{i}^{(n)}(\mathbf{x}, \mathbf{y})=N_{k l i}^{(n)}(\mathbf{y}) u_{k, l}^{(n-1)}+\Pi_{i l}^{(n)}(\mathbf{y}) \phi_{, l}^{(n-1)}$,

$\phi^{(n)}(\mathbf{x}, \mathbf{y})=\Psi_{k l}^{(n)}(\mathbf{y}) u_{k, l}^{(n-1)}+\Theta_{l}^{(n)}(\mathbf{y}) \phi_{, l}^{(n-1)}$,

where $\mathbf{N}^{(n)}, \boldsymbol{\Pi}^{(n)}, \Psi^{(n)}$ and $\boldsymbol{\Theta}^{(n)}$ are Y-periodic continuous functions and for their uniqueness, the functions satisfy $\left\langle\mathbf{N}^{(n)}\right\rangle=\left\langle\boldsymbol{\Pi}^{(n)}\right\rangle=\left\langle\Psi^{(n)}\right\rangle=$ $\left\langle\boldsymbol{\Theta}^{(n)}\right\rangle=\mathbf{0}$.

Substituting (29)-(30) into (27)-(28) (contribution $\varepsilon^{-1}$ ), we have

$-L_{i m n p} u_{m, n p}^{(0)}+L_{i m n} u_{m, n}^{(0)}+P_{i m} \phi_{, m}^{(0)}-P_{i m n} \phi_{, m n}^{(0)}=0$,

$Q_{m n p} u_{m, n p}^{(0)}+Q_{m n} u_{m, n}^{(0)}+R_{m} \phi_{, m}^{(0)}-R_{m n} \phi_{, m n}^{(0)}=0$,

where the coefficients $\mathbf{L}, \mathbf{P}, \mathbf{Q}$ and $\mathbf{R}$ involve derivatives with respect to the rapid variable $\mathbf{y}$ of the local functions $\mathbf{N}^{(n)}, \boldsymbol{\Pi}^{(n)}, \Psi^{(n)}$ and $\Theta^{(n)}$. Notice that the coefficients with higher orders $L_{i m n}, P_{i m n}, Q_{m n p}$ and $R_{m n}$ are associated with the flexoelectric tensor $\boldsymbol{\mu}$; on the other hand, the remaining coefficients $L_{i m n}, P_{i m}, Q_{m n}$ and $R_{m}$ are related to stiffness, piezoelectric and dielectric tensors. See the derivation in Appendix B Eqs. (B.7)-(B.8). 


\subsection{Local problems}

The local function $\mathbf{N}^{(n)}, \mathbf{\Pi}^{(n)}, \Psi^{(n)}$ and $\Theta^{(n)}$ are necessary to obtain the expressions of $\hat{\sigma}_{i j}$ and $\hat{D}_{i}$ of the homogenized problem (25)-(26).

Substituting (29)-(30) into (20) the following equations are derived

$\mu_{l i j k} \varrho_{r, l} \varrho_{s, k} \Psi_{k l \mid r s}^{(1)} u_{k, l}^{(0)}+\mu_{l i j k} \varrho_{r, l} \varrho_{s, k} \Theta_{l \mid r s}^{(1)} \phi_{l}^{(0)}=0$,

$\mu_{i k j l} \varrho_{r, l} \varrho_{s, j} N_{i l k \mid r s}^{(1)} u_{i, l}^{(0)}+\mu_{i k j l} \varrho_{r, l} \varrho_{s, j} \Pi_{k l \mid r s}^{(1)} \phi_{l}^{(0)}=0$.

Considering the linear independence between the functions $u_{k, l q}^{(0)}, u_{k, l}^{(0)}$, $\phi_{, l}^{(0)}$ and $\phi_{, l j}^{(0)}$, the continuity and the periodicity of the local functions, we can concluded that $\Psi_{m n \mid r s}^{(1)}=\Theta_{m \mid r s}^{(1)}=N_{n m k \mid r s}^{(1)}=\Pi_{m \mid r s}^{(1)}=0$. Moreover, due to the above mentioned linear independence, the coefficients of (31)(32) are identically zero.

The local functions $\Psi_{m n}^{(1)}, \Psi_{m n p}^{(2)}, N_{m n k}^{(1)}, N_{m n l k}^{(2)}, \Theta_{m}^{(1)}, \Theta_{m n}^{(2)}, \Pi_{k l}^{(1)}, \Pi_{l m k}^{(2)}$ are the solutions of the following local problems

$L_{i m n p} \equiv\left(\varrho_{t, l}\left(\mu_{p i l j}+\mu_{j i l p}\right) \varrho_{s, j} \Psi_{m n \mid s}^{(1)}+\varrho_{t, l} \mu_{k i l j} \varrho_{s, j} \varrho_{r, k} \Psi_{m n p \mid r s}^{(2)}\right)_{\mid t}=0$,

$L_{i m n} \equiv\left(\varrho_{t, j} C_{i j m n}+\varrho_{r, l} C_{i j k l} \varrho_{t, j} N_{m n k \mid r}^{(1)}+\varrho_{t, j} e_{k i j} \varrho_{r, k} \Psi_{m n \mid r}^{(1)}\right)_{\mid t}=0$,

$Q_{m n p} \equiv \varrho_{t, i}\left(\mu_{i m n p}+\left(\mu_{i k p l}+\mu_{i k l p}\right) \varrho_{r, l} N_{m n k \mid r}^{(1)}+\mu_{i k l j} \varrho_{r, l} \varrho_{s, j} N_{m n p k \mid r s}^{(2)}\right)_{\mid t}=0$,

$Q_{m n} \equiv\left(\varrho_{t, i} e_{i m n}+\varrho_{t, i} e_{i k l} \varrho_{r, k} N_{m n l \mid r}^{(1)}-\varrho_{t, i} \kappa_{i j} \varrho_{s, j} \Psi_{m n \mid s}^{(1)}\right)_{\mid t}=0$,

$P_{i m n} \equiv\left(\varrho_{t, l} \mu_{m i l n}+\varrho_{t, l}\left(\mu_{n i l j}+\mu_{n i j l}\right) \varrho_{s, j} \Theta_{m \mid s}^{(1)}+\varrho_{t, l} \mu_{k i l j} \varrho_{r, k} \varrho_{s, j} \Theta_{m n \mid r s}^{(2)}\right)_{\mid t}=0$,

$P_{i m} \equiv\left(\varrho_{t, j} e_{m i j}+e_{k i j} \varrho_{r, k} \varrho_{t, j} \Theta_{m \mid r}^{(1)}+\varrho_{t, j} C_{i j k l} \varrho_{r, l} \Pi_{m k \mid r}^{(1)}\right)_{\mid t}=0$,

$R_{m} \equiv\left(\varrho_{t, j} e_{j k l} \varrho_{r, l} \Pi_{m k \mid r}^{(1)}-\varrho_{t, j} \kappa_{j m}-\varrho_{t, j} \kappa_{j k} \varrho_{r, k} \Theta_{m \mid r}^{(1)}\right)_{\mid t}=0$,

$R_{l m} \equiv\left(\varrho_{t, i}\left(\mu_{i k p m}+\mu_{i k m p}\right) \varrho_{r, p} \Pi_{k l \mid r}^{(1)}+\varrho_{t, i} \mu_{i k p n} \varrho_{r, n} \varrho_{s, p} \Pi_{l m k \mid r s}^{(2)}\right)_{\mid t}=0$.

and satisfy the continuity conditions at the interface given in (B.12)(B.21).

Solving the $L Q$ (35)-(38) and $P R$ (39)-(42) systems, the local functions are obtained. As a particular case, the $L Q$ and $P R$ problems for one-dimensional flexoelectric rod are deduced in [24] (Eqs. (13)-(20)).

\subsection{Effective coefficients and homogenized problem}

Finally, the expressions of the effective stress $\hat{\sigma}_{i j}$ and the effective electric displacement $\hat{D}_{i}$ from the Eqs. (25)-(26), can be written in terms of the effective coefficients as follows

$\hat{\sigma}_{i j}=\hat{C}_{i j k l} u_{k, l}^{(0)}+\hat{e}_{k i j} \phi_{, k}^{(0)}-\hat{\mu}_{l i j k} \phi_{, k l}^{(0)}$,

$\hat{D}_{i}=\hat{e}_{i k l} u_{k, l}^{(0)}+\hat{\mu}_{i j k l} u_{j, k l}^{(0)}-\hat{\kappa}_{i j} \phi_{, j}^{(0)}$,

where

$\hat{C}_{i p m n}=\left\langle C_{i p m n}+C_{i p k l} \varrho_{r, l} N_{m n k \mid r}^{(1)}+e_{k i p} \varrho_{r, k} \Psi_{m n \mid r}^{(1)}\right\rangle$,

$\hat{\mu}_{m i p n}=\left\langle\mu_{m i p n}+\mu_{k i p j} \varrho_{s, j} \varrho_{r, k} \Theta_{m k \mid r s}^{(2)}+\left(\mu_{n i p k}+\mu_{k i p n}\right) \varrho_{r, k} \Theta_{m \mid r}^{(1)}\right\rangle$,

$\hat{\mu}_{m i p n}=\left\langle\mu_{m i p n}+\left(\mu_{m k n l}+\mu_{m k l n}\right) \varrho_{r, l} N_{i p k \mid r}^{(1)}+\mu_{m k l j} \varrho_{s, j} \varrho_{r, l} N_{i p n k \mid s r}^{(2)}\right\rangle$,

$\hat{e}_{\min }=\left\langle e_{\min }+e_{k i n} \varrho_{r, k} \Theta_{m \mid r}^{(1)}+C_{i n k l} \varrho_{r, l} \Pi_{m k \mid r}^{(1)}\right\rangle$

$\hat{e}_{\min }=\left\langle e_{\min }+e_{m k l} \varrho_{r, l} N_{i n k \mid r}^{(1)}-\kappa_{p j} \varrho_{r, k} \Psi_{i n \mid r}^{(1)}\right\rangle$, $\hat{\kappa}_{m n}=\left\langle\kappa_{m n}+\kappa_{n k} \varrho_{r, k} \Theta_{m \mid r}^{(1)}-e_{n k l} \varrho_{r, l} \Pi_{m k \mid r}^{(1)}\right\rangle$.

The expression (45) is known as the effective stiffness tensor. The Eq. (48) represent the effective piezoelectric tensor. In addition, (49) is called the permittivity tensor and finally (46) and (47) denote the effective flexoelectric tensor.

The equality of the equations of the piezoelectric tensor (48) is obtained by the solution of the $L Q$ problems (36), (38) and the $P R$ problems (40), (42), see Lemma 4.4 of [25].

Substituting the Eqs. (43)-(44) into (25)-(26), the homogenized problem associated to (10)-(14), takes the following form

$\left(\hat{C}_{i j k l} u_{k, l}^{(0)}+\hat{e}_{k i j} \phi_{, k}^{(0)}-\hat{\mu}_{l i j k} \phi_{, l k}^{(0)}\right)_{, j}+f_{i}=0$,

$\left(\hat{e}_{i k l} u_{k, l}^{(0)}+\hat{\mu}_{i j k l} u_{j, k l}^{(0)}-\hat{\kappa}_{i j} \phi_{, j}^{(0)}\right)_{, i}=0$,

with boundary conditions

$u_{i}^{(0)}=u_{i}^{0}$, on $\partial \Omega_{u}, \quad \hat{t}_{i}=t_{i}^{0}$, on $\partial \Omega_{t}$,

$u_{i, j}^{(0)} n_{j}=s_{i}^{0}$, on $\partial \Omega_{s}, \quad \hat{\mu}_{l i j k} \hat{E}_{l} n_{i} n_{j}=r_{i}^{0}$, on $\partial \Omega_{r}$,

$\phi^{(0)}=\phi^{0}$, on $\partial \Omega_{\phi}, \quad \hat{D}_{i} n_{i}=-\tau$, on $\partial \Omega_{\tau}$,

where $\hat{t}_{i}, \hat{E}_{l}, \hat{D}_{i}$ are the effective traction, electric field and electrical displacement respectively.

\section{Numerical calculations}

In this section, firstly, the model is validated, computing the effective properties of the structure presented in [22]. The intention is to verify the accuracy of the methodology described in Section 3, considering piezoelectric laminated composites PZT-5A/Araldite (Table 1) as limit case of flexoelectric materials. Now, two different cases of flexoelectric laminated media are considered. Case 1: rectangular bi-laminated composite with flexoelectric components is analyzed. The materials of the layers are Barium Titanate $\left(\mathrm{BaTiO}_{3}\right)$ and Gallium Arsenide (GaAs), see Table 2. Finally, Case 2: a flexoelectric wavy-laminated structure made of two layers of Barium Titanate $\left(\mathrm{BaTiO}_{3}\right)$ and Gallium Arsenide (GaAs) is studied.

All numerical examples presented in the following sections are particular cases of stratified composites, i.e. the function $\varrho: \mathbb{R}^{n} \rightarrow \mathbb{R}[17]$. Therefore, the local problems are solved using the description given in Appendix D.

\subsection{Limit case: piezoelectric non-wavy laminated composite}

Piezoelectric structures are particular cases of flexoelectric materials when $\boldsymbol{\mu}=\mathbf{0}$. To validate the method presented in this work, a bilaminated piezocomposite material is studied. The constituents of the structure are PZT-5A and Araldite with volume fractions $V_{1}$ and $V_{2}$, respectively. The mechanical properties of these materials are given in Table 2. As it is described in [22], the layers are considered transversal to the $x_{3}$ axis, i.e. $\rho\left(x_{1}, x_{2}, x_{3}\right)=x_{3}$ and $y=x_{3} / \varepsilon$, see Fig. $1 \mathrm{a}$.

The effective properties of the bi-laminate piezocomposite are estimated using two different methods: the Eqs. (45), (48), (49) (Present model) and the approach described in [22]. In Fig. 2, a comparison of the effective coefficients $\hat{C}_{2222}, \hat{e}_{113}, \hat{\kappa}_{11}$ and $\hat{\kappa}_{22}$ are shown. The numerical results are computed using the two methods for different volume fractions. The dashed lines represent the coefficients derived from the present model (AHM) and the circles are the effective properties obtained from Grekov et al. [22]. A good concordance between the two 
Table 1

Mechanical properties of the PZT-5A and Araldite, [22]. The parameters $C_{i j k l}, e_{i j k}, \kappa_{i j} / \kappa_{0}$ are the constituents of the stiffness $(\mathrm{GPa})$, piezoelectric $\left(\mathrm{C} / \mathrm{m}^{2}\right)$ and relative permittivity tensors, respectively.

\begin{tabular}{lllllllllll}
\hline Parameters & $C_{1111}$ & $C_{1122}$ & $C_{1133}$ & $C_{3333}$ & $C_{2323}$ & $e_{311}$ & $e_{333}$ & $e_{313}$ & $\kappa_{11} / \kappa_{0}$ & $\kappa_{33} / \kappa_{0}$ \\
\hline PZT-5A & 121 & 75.4 & 75.2 & 111 & 21.1 & -5.4 & 15.8 & 12.3 & 916 & 830 \\
Araldite & 5.46 & 2.94 & 2.94 & 5.46 & 1.26 & 0 & 0 & 0 & 7.0 & 7.0 \\
\hline
\end{tabular}

Table 2

Mechanical properties of $\mathrm{BaTiO}_{3}$ and GaAs. The parameters $C_{i j k l}, e_{i j k}, \kappa_{i j} / \kappa_{0}$ and $\mu_{i j k l}$ are the constituents of the stiffness $(\mathrm{GPa})$, piezoelectric $\left(\mathrm{C} / \mathrm{m}^{2}\right)$, relative permittivity and flexoelectric $(\mathrm{nC} / \mathrm{m})$ tensors, respectively.

\begin{tabular}{lllllllll}
\hline Parameters & $C_{1111}$ & $C_{1122}$ & $C_{2323}$ & $e_{123}$ & $\kappa_{11} / \kappa_{0}$ & $\mu_{1111}$ & $\mu_{1221}$ & $\mu_{1212}$ \\
\hline BaTiO $_{3}[13]$ & 358.1 & 115.2 & 149.8 & 0 & 1408 & 0.15 & -5.46 & 1.90 \\
GaAs $[26,27]_{118.8}$ & 53.8 & 59.4 & -0.16 & 10.99 & 0.514 & -0.838 & 0.265 \\
\hline
\end{tabular}

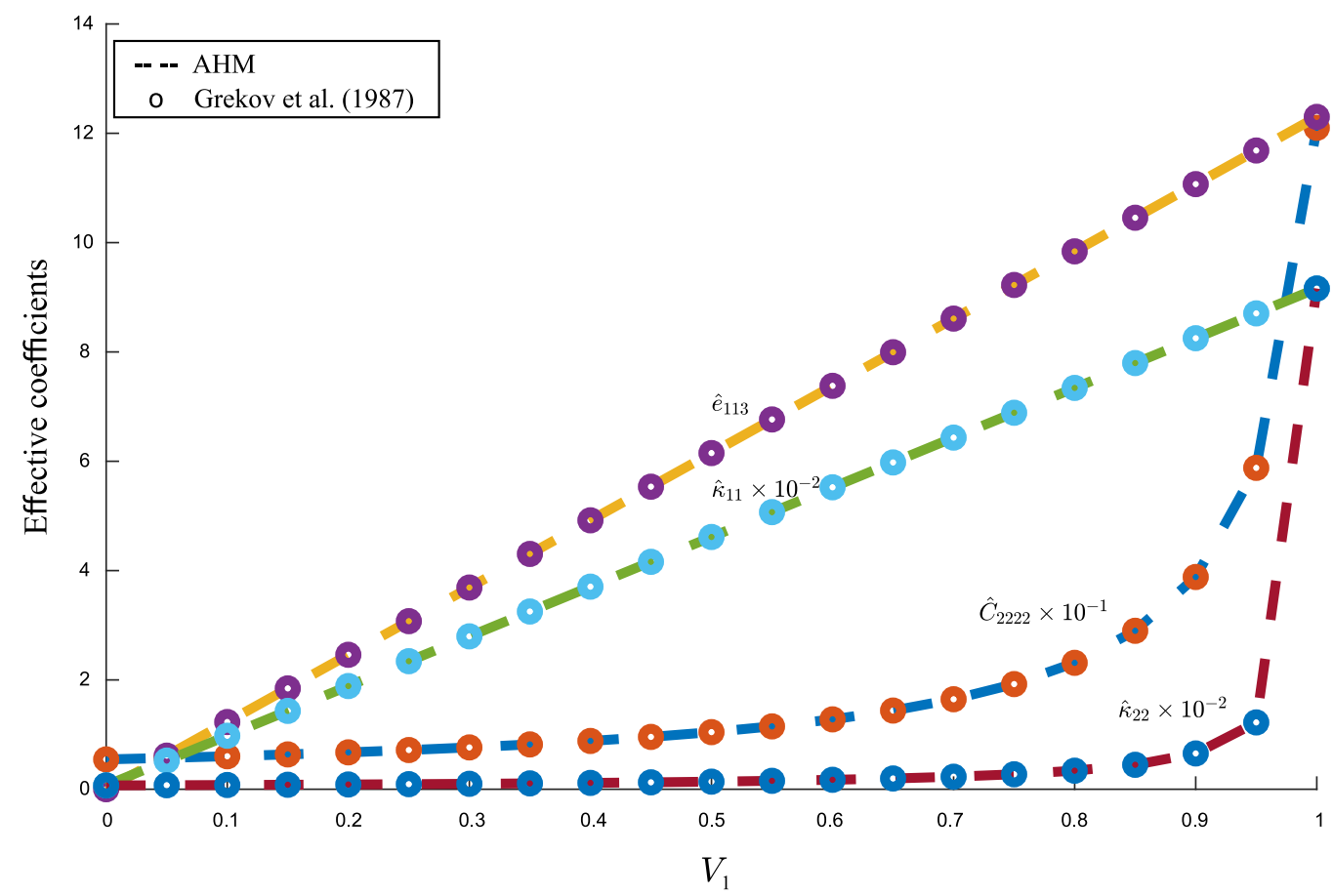

Fig. 2. Comparison of the effective coefficients $C_{2222}, e_{113}, \kappa_{11}$ and $\kappa_{22}$ obtained using the formulas (45), (48),(49) and the model described in [22].

methods is appreciated. Finally, this example validates the procedure presented in Section 3, taking the piezocomposites as a limit case of flexoelectric structures.

\subsection{Case 1: non-wavy laminated flexoelectric composite}

In this section a flexoelectric rectangular multilayered composite is proposed, where the layers are transversal to the axis $x_{3}$, Fig. 1 a. For this particular case, the fast variation of the properties occurs in the $x_{3}$ direction, i.e. $y=x_{3} / \varepsilon$, where $\rho\left(x_{1}, x_{2}, x_{3}\right)=x_{3}$, see [23]. The constituents are two cubic symmetric materials, Barium Titanate $\left(\mathrm{BaTiO}_{3}\right)$ and Gallium Arsenide (GaAs), with volume fractions $V_{1}=V_{2}=0.5$. The materials properties are given in Table 2.

Solving the $L Q$ (35)-(38) and $P R$ (39)-(42) systems and substituting the solution into (45)-(49), the effective coefficients are computed. The average operator for a laminate composite takes the form $\langle\bullet\rangle=0.5(\bullet)^{(1)}+$ $0.5(\bullet)^{(2)}$. The obtained effective coefficient matrices $\hat{\mathbf{C}}_{6 \times 6} \hat{\mathbf{e}}_{3 \times 6}, \hat{\boldsymbol{\kappa}}_{3 \times 3}$ and $\hat{\boldsymbol{\mu}}_{6 \times 9}$ are given as follows

$\hat{\mathbf{C}}_{6 \times 6}=\left(\begin{array}{cccccc}234.497 & 80.547 & 69.095 & 0 & 0 & 0 \\ 80.547 & 234.497 & 69.095 & 0 & 0 & 0 \\ 69.095 & 69.095 & 178.412 & 0 & 0 & 0 \\ 0 & 0 & 0 & 85.068 & 0 & 0 \\ 0 & 0 & 0 & 0 & 85.068 & 0 \\ 0 & 0 & 0 & 0 & 0 & 104.600\end{array}\right)$

$\hat{\mathbf{e}}_{3 \times 6}=\left(\begin{array}{cccccc}0 & 0 & 0 & -0.090 & 0 & 0 \\ 0 & 0 & 0 & 0 & -0.090 & 0 \\ 0 & 0 & 0 & 0 & 0 & -0.159\end{array}\right)$,

$\hat{\boldsymbol{\kappa}}_{3 \times 3}=\left(\begin{array}{ccc}709.495 & 0 & 0 \\ 0 & 709.495 & 0 \\ 0 & 0 & 21.8081\end{array}\right)$, 

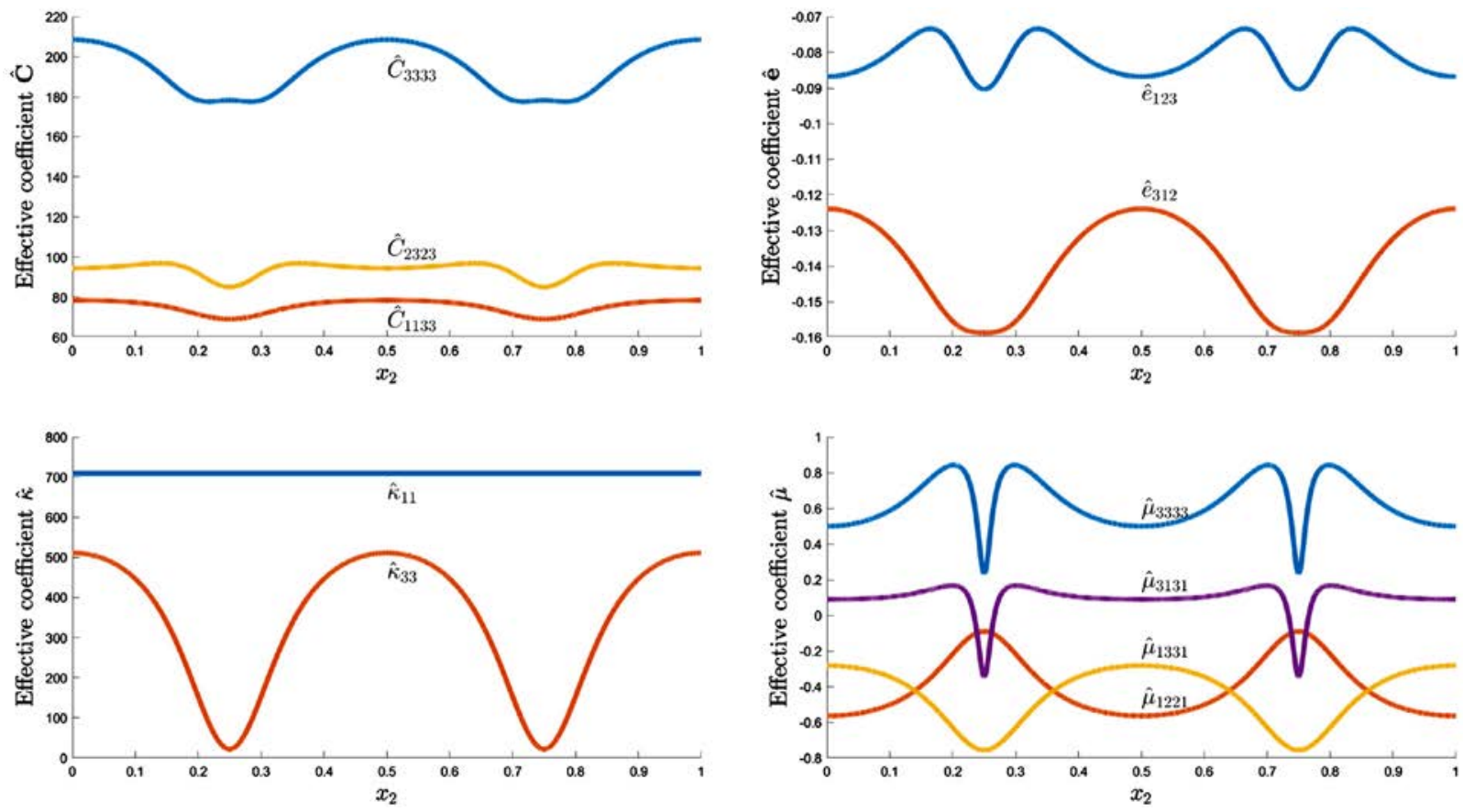

Fig. 3. Effective coefficients components $\hat{C}_{3333}, \hat{C}_{2323}, \hat{C}_{1133}$ (stiffness), $\hat{e}_{123}, \hat{e}_{312}$ (piezoelectric), $\hat{\kappa}_{11}, \hat{\kappa}_{33}$ (relative effective permittivity) and $\hat{\mu}_{3333}, \hat{\mu}_{3131}, \hat{\mu}_{1331}, \hat{\mu}_{1221}$ (flexoelectric).

$\hat{\boldsymbol{\mu}}_{6 \times 9}=\left(\begin{array}{ccccccc}0.334 & 0 & 0 & 0 & -0.090 & 0 & 0 \\ -0.090 & 0 & 0 & 0 & 0.334 & 0 & 0 \\ -0.757 & 0 & 0 & 0 & -0.757 & 0 & 0 \\ 0 & 0 & 0 & 0 & 0 & -0.346 & 0 \\ 0 & 0 & -0.346 & 0 & 0 & 0 & -0.184 \\ 0 & 0.495 & 0 & 0.495 & 0 & 0 & 0\end{array}\right.$

Notice that the constituents of the original heterogeneous body are two cubic symmetric layers, however the global properties of the homogenized solid after homogenization process have a tetragonal $(\overline{4} 2 \mathrm{~m})$ symmetry. The structure of the matrices $\hat{\mathbf{C}}_{6 \times 6}, \hat{\mathbf{e}}_{3 \times 6}$ and $\hat{\boldsymbol{\kappa}}_{3 \times 3}$ are characterized in Figure 3.14 of [26] and for the flexoelectric contribution tensor, the matrix $\hat{\boldsymbol{\mu}}_{6 \times 9}$ has the same linearly independent components as it is shown in the appendix of [28].

Notice that the effective coefficients for the particular case of rectangular laminate composites with a fixed volume fraction are constant functions.

\subsection{Case 2: wavy flexoelectric composite}

In order to point out the influence of the composite geometry into the effective properties, a two-element wavy laminate composite is studied as it is illustrated in Fig. 1b. The stratification function used to describe the undulating layers is given by

$\varrho\left(x_{1}, x_{2}, x_{3}\right)=x_{3}-\frac{1}{4} \sin \left(2 \pi x_{2}\right)$,

and the corresponding gradient is

$\frac{\partial \varrho}{\partial x_{1}}=0, \quad \frac{\partial \varrho}{\partial x_{2}}=-\frac{\pi}{2} \cos \left(2 \pi x_{2}\right) \quad \frac{\partial \varrho}{\partial x_{3}}=1$.

The constituents of the bi-layered composite are the cubic symmetric materials Barium Titanate $\left(\mathrm{BaTiO}_{3}\right)$ and Gallium Arsenide (GaAs). The properties of the layers are presented in Table 2. The local problems

$\left.\begin{array}{cc}\hline 0 & -2.286 \\ 0 & -2.286 \\ 0 & 0.232 \\ -0.184 & 0 \\ 0 & 0 \\ 0 & 0\end{array}\right)$

(35)-(42) are solved for the case of a stratified composite, following the approach described in Appendix D. The expressions of the local functions are substituted in (45)-(49) to compute the effective coefficients $\hat{\mathbf{C}}, \hat{\mathbf{e}}, \hat{\boldsymbol{\kappa}}$ and $\hat{\boldsymbol{\mu}}$.

To illustrate the behavior of the effective coefficients of the studied wavy composite, some non-zero components of the effective tensors are computed. In Fig. 3, the effective components $\hat{C}_{3333}, \hat{C}_{2323}, \hat{C}_{1133}$ (stiffness), $\hat{e}_{123}, \hat{e}_{312}$ (piezoelectric), $\hat{\kappa}_{11}, \hat{\kappa}_{33}$ (relative effective permittivity) and $\hat{\mu}_{3333}, \hat{\mu}_{3131}, \hat{\mu}_{1331}, \hat{\mu}_{1221}$ (flexoelectric) tensors are shown, considering the volume fraction $V_{1}=V_{2}=0.5$. Distinctly for the rectangular case, where all the effective coefficients are constants along the medium, for the wavy laminate structure, the effective coefficients are variable and depend on the position throughout the composite [17]. It can be seen that the average properties expressions (45)-(49) are related to the gradient of the stratification function (59). Thus, the overall tensors for this particular example are smooth continuous functions and 1 -periodic respect to $x_{2}$. Due to the continuity of the coefficients, the homogenized problem (50)-(54) is easier to solve compared with the heterogeneous problem, where the coefficients are rapidly oscillating discontinuous functions.

Although many methods offer efficient strategies and techniques for calculating effective coefficients, such as FEM $[29,30]$, the asymptotic homogenization method is an important tool for calculating effective properties. First of all, the equations presented in this manuscript are explicit expressions and they are easy to compute. The local problems are partial differential equations, but for the particular case of multilayered composites, the local problems are reduced to systems of linear algebraic equations and they can be solved analytically. On the other hand, 
the formulas obtained are not limited to the case of wavy structures; the calculations were made with a general $\boldsymbol{\rho}$ function, which allows adapting the formulas to different geometries, including more general cases of composites such as quasi-periodic structures. The computational cost of programming the effective coefficients is low compared with other similar methods, such as FEM. Finally, these expressions allow to calibrate numerical algorithms as reflected for FEM in [31].

\section{Conclusions}

The manuscript offers an extended study to find the effective coefficients of flexoelectric composite with complex geometry. The equilibrium problem for a flexoelectric structure is derived for the case of three-dimensional solid. In this work, the symmetry of the flexoelectric tensor is taken into account, as a low symmetry tensor, due to the gradient term of its definition. The matrix representation and symmetry of the flexoelectric tensor are provided.

The validation with the results reported in the literature for piezoelectric materials provides reliability to extend the two scales asymptotic homogenization method to the case of flexoelectric composites. The AHM is used to find the effective material properties. The manuscript presents a step-by-step strategy to derive the local problems and the effective coefficients equations for structures with generalized periodicity. The existence and uniqueness of the solution of the local problems are guaranteed. Following the proposal, the homogenized problem for three-dimensional flexoelectric composites is derived.

Bi-materials rectangular and wavy laminated composites are considered. A comparison between the effective properties of a rectangular and a wavy media provides important outcomes about the behavior of the coefficients of the homogenized problem. In the case of rectangular multi-layered composites, the global properties are constant throughout the structure, however, in the case of wavy structures, the average properties are continuous functions that depend on the stratifying function. The numerical results yield information about the flexoelectric effect for future research, as well as its applications, for example, in the regeneration of bone tissues.

This method is an extension of the procedure presented in previous mentioned works to flexoelectric composites with perfect contact at the interface. It can be extended to other types of composite with different interface contact conditions. Several applications in engineering, biomechanics can be considered due to the broad spectrum of composites covered by the present approach.

\section{Declaration of Competing Interest}

The authors declare that they have no known competing financial interests or personal relationships that could have appeared to influence the work reported in this paper.

\section{Appendix A. Flexoelectric tensor}

The ambiguity in the definition of the flexoelectric tensor has led several authors to adopt different constitutive equations and therefore different versions of the flexoelectric tensor have been studied [32]. But all authors agree that the flexoelectric tensor is a low symmetry fourthorder tensor [28]. The general expression of the flexoelectric tensor has 54 linearly independent components. This leads to consider a symmetry of the tensor with respect to two of the indices, in this work we have assumed $\mu_{i j k l}=\mu_{i k j l}$, the same symmetry is taken in [13].

The equilibrium Eqs. (1)-(5) are reported in [13] for a flexoelectric structure, where the tensor $\boldsymbol{\mu}$ is combined from the direct and converse flexoelectric tensors [1]. A matrix representation of the constitutive re- lations (6)-(7) is

$\left[\begin{array}{l}\boldsymbol{\sigma}_{6 \times 1} \\ \mathbf{D}_{3 \times 1}\end{array}\right]=\left[\begin{array}{cccc}\mathbf{C}_{6 \times 6} & -\mathbf{e}_{6 \times 3}^{T} & \mathbf{0}_{6 \times 18} & \boldsymbol{\mu}_{6 \times 9} \\ \mathbf{e}_{3 \times 6} & \boldsymbol{\kappa}_{3 \times 3} & \boldsymbol{\mu}_{3 \times 18} & \mathbf{0}_{3 \times 9}\end{array}\right]\left[\begin{array}{c}\boldsymbol{\epsilon}_{6 \times 1} \\ \mathbf{E}_{3 \times 1} \\ \nabla \boldsymbol{\epsilon}_{18 \times 1} \\ \nabla \mathbf{E}_{9 \times 1}\end{array}\right]$,

where

$\sigma_{6 \times 1}=\left[\sigma_{11}, \sigma_{22}, \sigma_{33}, \sigma_{23}, \sigma_{13}, \sigma_{12}\right]^{T}$,

$\mathbf{D}_{3 \times 1}=\left[D_{1}, D_{2}, D_{3}\right]^{T}$,

$\boldsymbol{\epsilon}_{6 \times 1}=\left[\epsilon_{11}, \epsilon_{22}, \epsilon_{33}, 2 \epsilon_{23}, 2 \epsilon_{13}, 2 \epsilon_{12}\right]^{T}$,

$\mathbf{E}_{3 \times 1}=\left[E_{1}, E_{2}, E_{3}\right]^{T}$,

$\nabla \epsilon_{18 \times 1}=\left[\epsilon_{11,1}, \epsilon_{22,1}, \epsilon_{33,1}, 2 \epsilon_{23,1}, \ldots, 2 \epsilon_{12,3}\right]^{T}$,

$\nabla \mathbf{E}_{9 \times 1}=\left[E_{1,1}, E_{2,1}, E_{3,1}, \ldots, E_{3,3}\right]^{T}$.

The matrices $\mathbf{C}_{6 \times 6}, \mathbf{e}_{3 \times 6}$ and $\boldsymbol{\kappa}_{3 \times 3}$ are the classical matrices of the elastic, piezoelectric and permittivity tensors respectively [26]. Finally, the flexoelectric matrices $\boldsymbol{\mu}_{3 \times 18}, \boldsymbol{\mu}_{6 \times 9}$ are deduced following the approach described in [28] and yields

$\boldsymbol{\mu}_{3 \times 18}=\left[\mu_{i n}\right], \quad i=1,2,3 ; \quad n=1,2,3, \ldots, 18$,

where $n=1,2,3, \ldots, 18$ refers to the combinations of the indexes $j k l=$ $111,221,331,231,131,121,112, \ldots, 123$, i.e. $\mu_{i n}=\mu_{i j k l}$ as follows

\begin{tabular}{llllllllll}
\hline$\mu_{\text {in }}$ & $n=1$ & $n=2$ & $n=3$ & $n=4$ & $n=5$ & $n=6$ & $n=7$ & $n=8$ & $n=9$ \\
\hline$i=1$ & $\mu_{1111}$ & $\mu_{1221}$ & $\mu_{1331}$ & $\mu_{1231}$ & $\mu_{1131}$ & $\mu_{1121}$ & $\mu_{1112}$ & $\mu_{1222}$ & $\mu_{1332}$ \\
$i=2$ & $\mu_{2111}$ & $\mu_{2221}$ & $\mu_{2331}$ & $\mu_{2231}$ & $\mu_{2131}$ & $\mu_{2121}$ & $\mu_{2112}$ & $\mu_{2222}$ & $\mu_{2332}$ \\
$i=3$ & $\mu_{3111}$ & $\mu_{3221}$ & $\mu_{3331}$ & $\mu_{3231}$ & $\mu_{3131}$ & $\mu_{3121}$ & $\mu_{3112}$ & $\mu_{3222}$ & $\mu_{3332}$ \\
\hline$\mu_{\text {in }}$ & $n=10$ & $n=11$ & $n=12$ & $n=13$ & $n=14$ & $n=15$ & $n=16$ & $n=17$ & $n=18$ \\
\hline$i=1$ & $\mu_{1232}$ & $\mu_{1132}$ & $\mu_{1122}$ & $\mu_{1113}$ & $\mu_{1223}$ & $\mu_{1333}$ & $\mu_{1233}$ & $\mu_{1133}$ & $\mu_{1123}$ \\
$i=2$ & $\mu_{2232}$ & $\mu_{2132}$ & $\mu_{2122}$ & $\mu_{2113}$ & $\mu_{2223}$ & $\mu_{2333}$ & $\mu_{2233}$ & $\mu_{2133}$ & $\mu_{2123}$ \\
$i=3$ & $\mu_{3232}$ & $\mu_{3132}$ & $\mu_{3122}$ & $\mu_{3113}$ & $\mu_{3223}$ & $\mu_{3333}$ & $\mu_{3233}$ & $\mu_{3133}$ & $\mu_{3123}$ \\
\hline
\end{tabular}

The flexoelectric matrix (A.2) described in the above table can be split in three sub-matrices

$\boldsymbol{\mu}_{3 \times 18}=\left[\left[\boldsymbol{\mu}_{3 \times 6}^{(1)}\right]\left[\boldsymbol{\mu}_{3 \times 6}^{(2)}\right]\left[\boldsymbol{\mu}_{3 \times 6}^{(3)}\right]\right]$.

On the other hand, the matrix $\boldsymbol{\mu}_{6 \times 9}$ has the form

$\boldsymbol{\mu}_{6 \times 9}=\left[\mu_{p q}\right]^{T}$,

where $q=1,2,3,4,5,6$ refers to the middle indexes $j k=$ $11,22,33,23,13,12$ and $p=1,2,3,4, \ldots, 9$ refers to all possible combinations of the outer indexes $i l=11,21,31,12,22,32,13,23,33$, i.e. $\mu_{p q}=\mu_{i j k l}$ as follows

\begin{tabular}{llllllllll}
\hline$\left[\mu_{p q}\right]^{T}$ & $p=1$ & $p=2$ & $p=3$ & $p=4$ & $p=5$ & $p=6$ & $p=7$ & $p=8$ & $p=9$ \\
\hline$q=1$ & $\mu_{1111}$ & $\mu_{2111}$ & $\mu_{3111}$ & $\mu_{1112}$ & $\mu_{2112}$ & $\mu_{3112}$ & $\mu_{1113}$ & $\mu_{2113}$ & $\mu_{3113}$ \\
$q=2$ & $\mu_{1221}$ & $\mu_{2221}$ & $\mu_{3221}$ & $\mu_{1222}$ & $\mu_{2222}$ & $\mu_{3222}$ & $\mu_{1223}$ & $\mu_{2223}$ & $\mu_{3223}$ \\
$q=3$ & $\mu_{1331}$ & $\mu_{2331}$ & $\mu_{3331}$ & $\mu_{1332}$ & $\mu_{2332}$ & $\mu_{3332}$ & $\mu_{1333}$ & $\mu_{2333}$ & $\mu_{3333}$ \\
$q=4$ & $\mu_{1231}$ & $\mu_{2231}$ & $\mu_{3231}$ & $\mu_{1232}$ & $\mu_{2232}$ & $\mu_{3232}$ & $\mu_{1233}$ & $\mu_{2233}$ & $\mu_{3233}$ \\
$q=5$ & $\mu_{1131}$ & $\mu_{2131}$ & $\mu_{3131}$ & $\mu_{1132}$ & $\mu_{2132}$ & $\mu_{3132}$ & $\mu_{1133}$ & $\mu_{2133}$ & $\mu_{3133}$ \\
$q=6$ & $\mu_{1121}$ & $\mu_{2121}$ & $\mu_{3121}$ & $\mu_{1122}$ & $\mu_{2122}$ & $\mu_{3122}$ & $\mu_{1123}$ & $\mu_{2123}$ & $\mu_{3123}$ \\
\hline
\end{tabular}

Notice that the matrix (A.4) can be written in terms of the submatrices given in (A.3) as follows

$\boldsymbol{\mu}_{6 \times 9}=\left[\left[\boldsymbol{\mu}_{3 \times 6}^{(1)}\right]^{T}\left[\boldsymbol{\mu}_{3 \times 6}^{(2)}\right]^{T}\left[\boldsymbol{\mu}_{3 \times 6}^{(3)}\right]^{T}\right]$. 
In the case of flexoelectric materials with cubic and tetragonal $(\overline{4} 2 \mathrm{~m})$ symmetries, $[13,28]$, the matrix $\boldsymbol{\mu}_{6 \times 9}$ has the forms

Cubic:

$\boldsymbol{\mu}_{6 \times 9}=\left(\begin{array}{lllllllll}\mu_{11} & 0 & 0 & 0 & \mu_{15} & 0 & 0 & 0 & \mu_{15} \\ \mu_{15} & 0 & 0 & 0 & \mu_{11} & 0 & 0 & 0 & \mu_{15} \\ \mu_{15} & 0 & 0 & 0 & \mu_{15} & 0 & 0 & 0 & \mu_{11} \\ 0 & 0 & 0 & 0 & 0 & \mu_{46} & 0 & \mu_{46} & 0 \\ 0 & 0 & \mu_{46} & 0 & 0 & 0 & \mu_{46} & 0 & 0 \\ 0 & \mu_{46} & 0 & \mu_{46} & 0 & 0 & 0 & 0 & 0\end{array}\right)$,

(A.6)

Tetragonal $\overline{4} 2 \mathrm{~m}$ :

$$
\boldsymbol{\mu}_{6 \times 9}=\left(\begin{array}{lllllllll}
\mu_{11} & 0 & 0 & 0 & \mu_{15} & 0 & 0 & 0 & \mu_{19} \\
\mu_{15} & 0 & 0 & 0 & \mu_{11} & 0 & 0 & 0 & \mu_{19} \\
\mu_{31} & 0 & 0 & 0 & \mu_{31} & 0 & 0 & 0 & \mu_{39} \\
0 & 0 & 0 & 0 & 0 & \mu_{46} & 0 & \mu_{48} & 0 \\
0 & 0 & \mu_{46} & 0 & 0 & 0 & \mu_{48} & 0 & 0 \\
0 & \mu_{62} & 0 & \mu_{62} & 0 & 0 & 0 & 0 & 0
\end{array}\right) .
$$

\section{Appendix B. Asymptotic homogenization method}

Substituting (16)-(19) into the constitutive relations (6)-(8), the following expressions are derived

$$
\begin{gathered}
\sigma_{i j}^{(0)}(\mathbf{x}, \mathbf{y})+\varepsilon \sigma_{i j}^{(1)}(\mathbf{x}, \mathbf{y})+\varepsilon^{2} \sigma_{i j}^{(2)}(\mathbf{x}, \mathbf{y})+\ldots=C_{i j k l} \partial_{l}\left(u_{k}^{(0)}+\varepsilon u_{k}^{(1)}+\varepsilon^{2} u_{k}^{(2)}+\ldots\right) \\
+e_{k i j} \partial_{j}\left(\phi^{(0)}+\varepsilon \phi^{(1)}+\varepsilon^{2} \phi^{(2)}+\ldots\right)-\mu_{l i j k} \partial_{l} \partial_{k}\left(\phi^{(0)}+\varepsilon \phi^{(1)}+\varepsilon^{2} \phi^{(2)}+\ldots\right),
\end{gathered}
$$

$$
\begin{gathered}
D_{i}^{(0)}(\mathbf{x}, \mathbf{y})+\varepsilon D_{i}^{(1)}(\mathbf{x}, \mathbf{y})+\varepsilon^{2} D_{i}^{(2)}(\mathbf{x}, \mathbf{y})+\cdots=e_{i k l} \partial_{l}\left(u_{k}^{(0)}+\varepsilon u_{k}^{(1)}+\varepsilon^{2} u_{k}^{(2)}+\ldots\right) \\
+\mu_{i k j l} \partial_{l} \partial_{j}\left(u_{k}^{(0)}+\varepsilon u_{k}^{(1)}+\varepsilon^{2} u_{k}^{(2)}+\ldots\right)-\kappa_{i j} \partial_{j}\left(\phi^{(0)}+\varepsilon \phi^{(1)}+\varepsilon^{2} \phi^{(2)}+\ldots\right) .
\end{gathered}
$$

Grouping by power of $\varepsilon$ in the right-hand sides of (B.1)-(B.2) and considering the above mentioned total derivative operator, yields

$$
\begin{aligned}
& \sigma_{i j}^{(0)}(\mathbf{x}, \mathbf{y})+\varepsilon \sigma_{i j}^{(1)}(\mathbf{x}, \mathbf{y})+\varepsilon^{2} \sigma_{i j}^{(2)}(\mathbf{x}, \mathbf{y})+\ldots=\varepsilon^{-1}\left(\mu_{l i j k} \varrho_{r, l} \varrho_{s, k} \phi_{\mid r s}^{(1)}\right) \\
& +C_{i j k l} u_{k, l}^{(0)}+C_{i j k l} \rho_{r, l} u_{k \mid r}^{(1)}+e_{k i j} \phi_{, j}^{(0)}+e_{k i j} \rho_{r, j} \phi_{\mid j}^{(1)}-\mu_{l i j k} \phi_{, l k}^{(0)} \\
& -\left(\mu_{k i j l}+\mu_{l i j k}\right) \varrho_{r, l} \phi_{\mid r, k}^{(1)}-\mu_{l i j k} \varrho_{r, l} \rho_{s, k} \phi_{\mid r s}^{(2)} \\
& +\varepsilon\left(C_{i j k l} u_{k, l}^{(1)}+C_{i j k l} o_{r, l} u_{k \mid r}^{(2)}+e_{k i j} \phi_{, j}^{(1)}+e_{k i j} \varrho_{r, j} \phi_{\mid j}^{(2)}-\mu_{l i j k} \phi_{, l k}^{(1)}\right. \\
& \left.-\left(\mu_{k i j l}+\mu_{l i j k}\right) \varrho_{r, l} \phi_{\mid r, k}^{(2)}-\mu_{l i j k} \varrho_{r, l} \varrho_{s, k} \phi_{\mid r s}^{(3)}\right) \\
& +\varepsilon^{2}\left(C_{i j k l} u_{k, l}^{(2)}+C_{i j k l} \varrho_{r, l} u_{k \mid r}^{(3)}+e_{k i j} \phi_{, j}^{(2)}+e_{k i j} \rho_{r, j} \phi_{l j}^{(3)}-\mu_{l i j k} \phi_{, l k}^{(2)}\right. \\
& \left.-\left(\mu_{k i j l}+\mu_{l i j k}\right) \varrho_{r, l} \phi_{\mid r, k}^{(3)}-\mu_{l i j k} \varrho_{r, l} \varrho_{s, k} \phi_{\mid r s}^{(4)}\right) \\
& \vdots \\
& D_{i}^{(0)}(\mathbf{x}, \mathbf{y})+\varepsilon D_{i}^{(1)}(\mathbf{x}, \mathbf{y})+\varepsilon^{2} D_{i}^{(2)}(\mathbf{x}, \mathbf{y})+\ldots=\varepsilon^{-1}\left(\mu_{i k j l} \varrho_{r, l} \varrho_{s, j} u_{k \mid r s}^{(1)}\right) \\
& +e_{i k l} u_{k, l}^{(0)}+e_{i k l} \rho_{r, l} u_{k \mid r}^{(1)}-\kappa_{i j} \phi_{, j}^{(0)}-\kappa_{i j} \rho_{s, j} \phi_{\mid s}^{(1)}+\mu_{i k j l} u_{k, l j}^{(0)} \\
& +\left(\mu_{i k l j}+\mu_{i k j l}\right) \varrho_{r, l} u_{k, j \mid r}^{(1)}+\mu_{i k j l} \varrho_{r, l} \varrho_{s, j} u_{k \mid r s}^{(2)} \\
& +\varepsilon\left(e_{i k l} u_{k, l}^{(1)}+e_{i k l} \rho_{r, l} u_{k \mid r}^{(2)}-\kappa_{i j} \phi_{, j}^{(1)}-\kappa_{i j} \rho_{s, j} \phi_{\mid s}^{(2)}+\mu_{i k j l} u_{k, l j}^{(1)}\right. \\
& \left.+\left(\mu_{i k l j}+\mu_{i k j l}\right) \varrho_{r, l} u_{k, j \mid r}^{(2)}+\mu_{i k j l} \varrho_{r, l} \varrho_{s, j} u_{k \mid r s}^{(3)}\right) \\
& +\varepsilon^{2}\left(e_{i k l} u_{k, l}^{(2)}+e_{i k l} o_{r, l} u_{k \mid r}^{(3)}-\kappa_{i j} \phi_{, j}^{(2)}-\kappa_{i j} o_{s, j} \phi_{\mid s}^{(3)}+\mu_{i k j l} u_{k, l j}^{(2)}\right. \\
& \left.+\left(\mu_{i k l j}+\mu_{i k j l}\right) \varrho_{r, l} u_{k, j \mid r}^{(3)}+\mu_{i k j l} \varrho_{r, l} \varrho_{s, j} u_{k \mid r s}^{(4)}\right)
\end{aligned}
$$

Replacing the expansions (18) and (19) into the equilibrium problem (1)(2), the following is derived

$$
\begin{aligned}
& \varepsilon^{-1} \varrho_{k, j} \sigma_{i j \mid k}^{(0)}+\sigma_{i j, j}^{(0)}+\varrho_{k, j} \sigma_{i j \mid k}^{(1)}+\varepsilon\left(\sigma_{i j, j}^{(1)}+\varrho_{k, j} \sigma_{i j \mid k}^{(2)}\right) \ldots+f_{i}=0 \\
& \varepsilon^{-1} \varrho_{k, i} D_{i \mid k}^{(0)}+D_{i, i}^{(0)}+\varrho_{k, i} D_{i \mid k}^{(1)}+\varepsilon\left(D_{i, i}^{(1)}+\varrho_{k, i} D_{i \mid k}^{(2)}\right) \ldots=0
\end{aligned}
$$

Substituting (29)-(30) into (27)-(28) and regrouping in terms of the derivatives of $u_{k}^{(0)}$ and $\phi^{(0)}$, it yields

$$
\begin{aligned}
& u_{k, l q}^{(0)} \underbrace{\left(-\mu_{m i j n} \varrho_{t, j} \varrho_{r, m} \varrho_{s, n} \Psi_{q k l \mid r s}^{(2)}-\left(\mu_{m i j q}+\mu_{q i j m}\right) \varrho_{t, j} \varrho_{r, m} \Psi_{k l \mid r}^{(1)}\right)_{\mid t}}_{L_{i k l q}} \\
& +u_{k, l}^{(0)} \underbrace{\left(\varrho_{t, j} C_{i j k l}+\varrho_{t, j} C_{i j n m} \varrho_{r, m} N_{k l n \mid r}^{(1)}+\varrho_{t, j} e_{j i m} \varrho_{r, m} \Psi_{k l \mid r}^{(1)}\right)_{\mid t}}_{L_{i k l}} \\
& +\phi_{, l}^{(0)} \underbrace{\left(\varrho_{t, j} e_{j i l}+\varrho_{t, j} C_{i j n m} \varrho_{r, m} \Pi_{n l \mid r}^{(1)}+\varrho_{t, j} e_{j i m} \varrho_{r, m} \Theta_{l \mid r}^{(1)}\right)_{\mid t}}_{P_{i l}} \\
& -\phi_{, l k}^{(0)} \underbrace{\left(\varrho_{t, j} \mu_{l i j k}+\varrho_{t, j}\left(\mu_{m i j k}+\mu_{k i j m}\right) \varrho_{r, m} \Theta_{l \mid r}^{(1)}-\varrho_{t, j} \mu_{m i j n} \varrho_{r, m} \varrho_{s, n} \Theta_{k l \mid r s}^{(2)}\right)_{\mid t}}_{P_{i l k}}=0,
\end{aligned}
$$

$$
\begin{aligned}
& u_{k, l q}^{(0)} \underbrace{\left(\varrho_{t, i} \mu_{i k q l}+\varrho_{t, i}\left(\mu_{i n j l}+\mu_{i n l j}\right) \varrho_{r, j} N_{k q n \mid r}^{(1)}+\varrho_{t, i} \mu_{i m j p} \varrho_{r, p} \varrho_{s, j} N_{q k l m \mid r s}^{(2)}\right)_{\mid t}}_{Q_{k l q}} \\
& +u_{k, l}^{(0)} \underbrace{\left(\varrho_{t, i} e_{i k l}+\varrho_{t, i} e_{i m n} \varrho_{r, n} N_{k l m \mid r}^{(1)}-\varrho_{t, i} \kappa_{i j} \varrho_{s, j} \Psi_{k l \mid s}^{(1)}\right)_{\mid t}}_{Q_{k l}} \\
& +\phi_{, l}^{(0)} \underbrace{\left(-\varrho_{t, i} \kappa_{i l}+\varrho_{t, i} e_{i m l} \varrho_{r, l} \Pi_{k l \mid r}^{(1)}-\varrho_{t, i} \kappa_{i j} \varrho_{s, j} \Theta_{l \mid s}^{(1)}\right)_{\mid t}}_{R_{l}} \underbrace{(0)}_{R_{l j}} \underbrace{\left.\varrho_{t, i}\left(\mu_{i k p j}+\varrho_{t, i} \mu_{i k j p}\right) \varrho_{r, p} \Pi_{k l \mid r}^{(1)}+\varrho_{t, i} \mu_{i k p n} \varrho_{r, n} \varrho_{s, p} \Pi_{l j k \mid r s}^{(2)}\right)_{\mid t}}_{, l, i}=0,
\end{aligned}
$$

where

$$
\begin{array}{r}
N_{l m p n}^{(2)}=N_{k l n}^{(2)} N_{m p k}^{(1)}+\Pi_{n l}^{(2)} \Psi_{m p}^{(1)}, \\
\Pi_{l m n}^{(2)}=N_{k l n}^{(2)} \Pi_{k m}^{(1)}+\Pi_{n l}^{(2)} \Theta_{m}^{(1)}, \\
\Psi_{l m n}^{(2)}=\Psi_{k l}^{(2)} N_{m n k}^{(1)}+\Theta_{l}^{(2)} \Psi_{k n}^{(1)}, \\
\Theta_{n l}^{(2)}=\Psi_{k l}^{(2)} \Pi_{k n}^{(1)}+\Theta_{l}^{(2)} \Theta_{n}^{(1)} .
\end{array}
$$

Now, the asymptotic expansions (16)-(19) are substituted into the interface conditions (15), where the different order of $\varepsilon$ contributions are

$$
\begin{array}{r}
{\left[\left[u_{i}^{(n)}\right]\right]=0,\left[\left[\phi^{(n)}\right]\right]=0,} \\
{\left[\left[\sigma_{i j}^{(n)} n_{j}\right]\right]=0,\left[\left[D_{i}^{(n)} n_{i}\right]\right]=0 .}
\end{array}
$$

Moreover, the equations (29)-(30) are substituted into (21)-(22), and the resulting expressions in terms of the local functions $\mathbf{N}^{(n)}, \mathbf{\Pi}^{(n)}, \Psi^{(n)}$ and $\boldsymbol{\Theta}^{(n)}$ are replaced in (B.11). 
The continuity conditions at the interface of the local functions $\Psi_{m n}^{(1)}$, $\Psi_{m n p}^{(2)}, N_{m n k}^{(1)}, N_{m n l k}^{(2)}, \Theta_{m}^{(1)}, \Theta_{m n}^{(2)}, \Pi_{k l}^{(1)}, \Pi_{l m k}^{(2)}$ are obtained from the first two equations of (B.11) for $n=1,2$

$$
\begin{gathered}
{\left[\left[N_{k l i}^{(1)}\right]\right]=0,\left[\left[\Pi_{i l}^{(1)}\right]\right]=0,} \\
{\left[\left[\Psi_{k l}^{(1)}\right]\right]=0,\left[\left[\Theta_{l}^{(1)}\right]\right]=0,}
\end{gathered}
$$

$$
\begin{gathered}
{\left[\left[N_{m n l k}^{(2)}\right]\right]=0,\left[\left[\Pi_{l m k}^{(2)}\right]\right]=0} \\
{\left[\left[\Psi_{m n p}^{(2)}\right]\right]=0,\left[\left[\Theta_{m n}^{(2)}\right]\right]=0,}
\end{gathered}
$$

and the remaining two equations of (B.11), for $n=0$

$$
\begin{aligned}
& {\left[\left[\left(\left(\mu_{p i l j}+\mu_{j i l p}\right) \varrho_{s, j} \Psi_{m n \mid s}^{(1)}+\mu_{k i l j} \varrho_{s, j} \varrho_{r, k} \Psi_{m n p \mid r s}^{(2)}\right) n_{j}\right]\right]=0,} \\
& {\left[\left[\left(C_{i j m n}+\varrho_{r, l} C_{i j k l} N_{m n k \mid r}^{(1)}+e_{k i j} \varrho_{r, k} \Psi_{m n \mid r}^{(1)}\right) n_{j}\right]\right]=0,} \\
& {\left[\left[\left(\mu_{i m n p}+\left(\mu_{i k p l}+\mu_{i k l p}\right) \varrho_{r, l} N_{m n k \mid r}^{(1)}+\mu_{i k l j} \varrho_{r, l} \varrho_{s, j} N_{m n l k \mid r s}^{(2)}\right) n_{i}\right]\right]=0,} \\
& {\left[\left[\left(e_{i m n}+e_{i k l} \varrho_{r, k} N_{m n l \mid r}^{(1)}-\kappa_{i j} \varrho_{s, j} \Psi_{m n \mid s}^{(1)}\right) n_{i}\right]\right]=0,} \\
& {\left[\left[\left(\mu_{m i j n}+\left(\mu_{n i j l}+\mu_{n i l j}\right) \varrho_{s, l} \Theta_{m \mid s}^{(1)}+\mu_{k i j l} \varrho_{r, k} \varrho_{s, l} \Theta_{m n \mid r s}^{(2)}\right) n_{j}\right]\right]=0,} \\
& {\left[\left[\left(e_{m i j}+e_{k i j} \varrho_{r, k} \Theta_{m \mid r}^{(1)}+C_{i j k l} \varrho_{r, l} \Pi_{m k \mid r}^{(1)}\right) n_{j}\right]\right]=0,} \\
& {\left[\left[\left(e_{i k l} \varrho_{r, l} \Pi_{m k \mid r}^{(1)}-\kappa_{i m}-\kappa_{i k} \varrho_{r, k} \Theta_{m \mid r}^{(1)}\right) n_{i}\right]\right]=0,} \\
& {\left[\left[\left(\left(\mu_{i k p m}+\mu_{i k m p}\right) \varrho_{r, p} \Pi_{k l \mid r}^{(1)}+\mu_{i k p n} \varrho_{r, n} \varrho_{s, p} \Pi_{l m k \mid r s}^{(2)}\right) n_{i}\right]\right]=0 .}
\end{aligned}
$$

The expressions (B.12)-(B.21) are the interface conditions for the local functions $\Psi_{m n}^{(1)}, \Psi_{m n p}^{(2)}, N_{m n k}^{(1)}, N_{m n l k}^{(2)}, \Theta_{m}^{(1)}, \Theta_{m n}^{(2)}, \Pi_{k l}^{(1)}, \Pi_{l m k}^{(2)}$.

\section{Appendix C. Existence and uniqueness of the solution of the local problems}

Let's assume that $\Omega \subset \mathbb{R}^{3}$ is a three-dimensional open connected bounded domain with an infinitely smooth boundary $\partial \Omega$. The material functions are supposed to be infinitely differentiable, rapidly oscillating and $\varepsilon \mathbf{Y}$-periodic in the local variable $\mathbf{y}=\boldsymbol{\rho}(\mathbf{x}) / \varepsilon$ where $\varepsilon>0$ is the usual small geometric parameter, and $\mathbf{Y}$ is the so-called periodic cell. For each $\mathbf{x}=\left(x_{1}, x_{2}, x_{3}\right) \in \Omega$ the material functions are defined as the following $\varepsilon$-family of functions:

$\mathbf{C} \equiv \mathbf{C}(\mathbf{x}, \mathbf{y}), \mathbf{e} \equiv \mathbf{e}(\mathbf{x}, \mathbf{y})$,

$\kappa \equiv \kappa(\mathbf{x}, \mathbf{y}), \mu \equiv \mu(\mathbf{x}, \mathbf{y})$

The material functions satisfy the usual symmetry conditions given by (9). Let's consider the system of Eqs. (36) and (38) for each value of $m, n=1,2,3$. The system can be written in the matrix form as follows,

$\frac{\partial}{\partial y_{t}}\left(\mathbf{D} \frac{\partial}{\partial y_{r}} \mathbf{N}\right)=-\frac{\partial}{\partial y_{t}} \mathbf{b}$,

where

$\mathbf{N}=\left[\begin{array}{llll}N_{m n 1}^{(1)} & N_{m n 2}^{(1)} & N_{m n 3}^{(1)} & \Psi_{m n}^{(1)}\end{array}\right]^{T}$,

$\mathbf{D}=\left[\begin{array}{cccc}\varrho_{r, l} C_{1 j 1 l} \varrho_{t, j} & \varrho_{r, l} C_{1 j 2 l} \varrho_{t, j} & \varrho_{r, l} C_{1 j 3 l} \varrho_{t, j} & \varrho_{t, j} e_{l 1 j} \varrho_{r, l} \\ \varrho_{r, l} C_{2 j 1 l} \varrho_{t, j} & \varrho_{r, l} C_{2 j 2 l} \varrho_{t, j} & \varrho_{r, l} C_{2 j 3 l} \varrho_{t, j} & \varrho_{t, j} e_{l 2 j} \varrho_{r, l} \\ \varrho_{r, l} C_{3 j 1 l} \varrho_{t, j} & \varrho_{r, l} C_{3 j 2 l} \varrho_{t, j} & \varrho_{r, l} C_{3 j 3 l} \varrho_{t, j} & \varrho_{t, j} e_{l 3 j} \varrho_{r, l} \\ \varrho_{t, j} e_{j 1 l} \varrho_{r, l} & \varrho_{t, j} e_{j 2 l} \varrho_{r, l} & \varrho_{t, j} e_{j 3 l} \varrho_{r, l} & -\varrho_{t, j} \kappa_{j l} \varrho_{r, l}\end{array}\right]$, $\mathbf{b}=\left[\begin{array}{llll}\varrho_{t, j} C_{1 j m n} & \varrho_{t, j} C_{2 j m n} & \varrho_{t, j} C_{3 j m n} & \varrho_{t, j} e_{j m n}\end{array}\right]^{T}$.

Besides, we make the further assumption that there exist a constant $\xi>0$ such that, for any vector $\mathbf{a}=\left(a_{1}, a_{2}, a_{3}\right)$, and any third-order symmetric matrix $M=\left(M_{i j}\right)$

$C_{i j k l} M_{i j} M_{k l} \geq \xi M_{i j} M_{i j}, \quad \kappa_{i j} a_{i} a_{j} \geq \xi a_{i} a_{i}$.

Using the Cauchy-Schwarz inequality [33], it is shown that the matrix $D_{t r}$ satisfies the following

$\left\{\mathbf{D} \frac{\partial}{\partial t_{r}} \mathbf{N}, \frac{\partial}{\partial y_{t}} \mathbf{N}\right\} \geq \xi_{1}\left\{\frac{\partial}{\partial t_{r}} \mathbf{N}, \frac{\partial}{\partial y_{t}} \mathbf{N}\right\}$,

where $\xi_{1}>0$ and $\{\bullet, \bullet\}$ denotes the scalar product. Under the assumption that $\left\langle\partial \mathbf{b}_{t} / \partial y_{t}\right\rangle=0$, the Theorem 1, p. 346 of [21] guarantee the existence of a general solution in the form,

$\mathbf{N}=\mathbf{N}_{0}+\mathbf{c}$,

where $\left\langle\mathbf{N}_{0}\right\rangle=0$. On the other hand, considering the condition $\langle\mathbf{N}\rangle=0$, it turns out that $\mathbf{c} \equiv \mathbf{0}$, and the solution of the problem is unique, see Proposition 1 in [17].

Following a similar idea, the existence and uniqueness of the solution of the $L Q$ and $P R$ problems can be proved.

\section{Appendix D. Solution of the local problem for stratified composites}

As a particular example of the given methodology, a stratified composite is considered, i.e. the case of a function $\boldsymbol{\rho}: \mathbb{R}^{3} \rightarrow \mathbb{R}[17]$; then, the local variable $y \in \mathbb{R}$. Also, a methodology to solve the local problems (35)-(42) for a stratified composite is presented.

From the expressions (45)-(49), the functions $N_{m n k}^{(1)}, \Psi_{m n}^{(1)}, \Theta_{m}^{(1)}, \Pi_{m k}^{(1)}$ are not necessary for finding the effective coefficients; only the derivatives are sufficient to get (45)-(49). For that reason, the systems of partial differential equations $L Q$ and $P R$ can be reduced. Following this idea, a methodology described in [17] is extended for the case of stratified flexoelectric composites.

For the case of stratified composite, the local problems (36), (38) $(L Q)$ have the following expressions

$$
\begin{aligned}
& \frac{\partial}{\partial y}\left(\frac{\partial \varrho}{\partial x_{j}} C_{i j m n}+\frac{\partial \varrho}{\partial x_{l}} C_{i j k l} \frac{\partial \varrho}{\partial x_{j}} \frac{\partial N_{m n k}^{(1)}}{\partial y}+\frac{\partial \varrho}{\partial x_{j}} e_{k i j} \frac{\partial \varrho}{\partial x_{k}} \frac{\partial \Psi_{m n}^{(1)}}{\partial y}\right)=0 \\
& \frac{\partial}{\partial y}\left(\frac{\partial \varrho}{\partial x_{j}} e_{j m n}+\frac{\partial \varrho}{\partial x_{j}} e_{j k l} \frac{\partial \varrho}{\partial x_{l}} \frac{\partial N_{m n k}^{(1)}}{\partial y}-\frac{\partial \varrho}{\partial x_{j}} \kappa_{j k} \frac{\partial \varrho}{\partial x_{k}} \frac{\partial \Psi_{m n}^{(1)}}{\partial y}\right)=0 .
\end{aligned}
$$

For every value of $m, n=1,2,3$, the systems (D.1)-(D.2) are $4 \times 4$ systems. Taking into account that the average of the local functions is zero, the solution of the $L Q$ problems (D.1)-(D.2) satisfies the system

$\left[D_{a b}\right]_{4 \times 4} \mathbf{N}_{4 \times 1}=\lambda_{4 \times 1}-\mathbf{b}_{4 \times 1}$,

where

$$
\begin{aligned}
& \mathbf{N}=\left[\frac{\partial N_{m n 1}^{(1)}}{\partial y}, \frac{\partial N_{m n 2}^{(1)}}{\partial y}, \frac{\partial N_{m n 3}^{(1)}}{\partial y}, \frac{\partial \Psi_{m n}^{(1)}}{\partial y}\right]^{T}, \\
& D_{a b}=\frac{\partial \varrho}{\partial x_{l}} C_{a j b l} \frac{\partial \varrho}{\partial x_{j}}, \quad a, b=1,2,3, \\
& D_{a 4}=\frac{\partial \varrho}{\partial x_{j}} e_{k a j} \frac{\partial \varrho}{\partial x_{k}}, \quad a=1,2,3, \\
& D_{4 b}=\frac{\partial \varrho}{\partial x_{j}} e_{j b l} \frac{\partial \varrho}{\partial x_{l}}, \quad b=1,2,3, \\
& D_{44}=-\frac{\partial \varrho}{\partial x_{j}} \kappa_{j k} \frac{\partial \varrho}{\partial x_{k}}, \quad \\
& \mathbf{b}=\left[\frac{\partial \varrho}{\partial x_{j}} C_{1 j m n}, \frac{\partial \varrho}{\partial x_{j}} C_{2 j m n}, \frac{\partial \varrho}{\partial x_{j}} C_{3 j m n}, \frac{\partial \varrho}{\partial x_{j}} e_{j m n}\right]^{T},
\end{aligned}
$$


and $\lambda$ satisfies the linear system

$\left\langle\left[D_{a b}\right]^{-1}\right\rangle_{4 \times 4} \lambda_{4 \times 1}=\left\langle\left[D_{a b}\right]^{-1} \mathbf{b}\right\rangle_{4 \times 1}$,

where $\langle\bullet\rangle$ denotes the average operator. This methodology is described in [17] for the case of wavy-laminated elastic composite materials. Similarly, the rest of the $L Q$ and $P R$ problems can be solved.

\section{CRediT authorship contribution statement}

D. Guinovart-Sanjuán: Investigation, Methodology, Software, Writing - original draft. K. Vajravelu: Conceptualization, Methodology, Investigation. R. Rodríguez-Ramos: Supervision, Writing - original draft, Writing - review \& editing, Formal analysis. R. Guinovart-Díaz: Writing - review \& editing, Software, Validation, Formal analysis. J. BravoCastillero: Conceptualization, Methodology. F. Lebon: Writing - review \& editing, Visualization. F.J. Sabina: Validation, Writing - review \& editing. J. Merodio: Resources, Validation.

\section{References}

[1] Sharma ND, Landis CM, Sharma P. Piezoelectric thin-film superlattices without using piezoelectric materials. J Appl Phys 2010;108(2):24304. doi:10.1063/1.3443404.

[2] Deng Q, Liu L, Sharma P. Flexoelectricity in soft materials and biological membranes. Journal of the Mechanics and Physics of Solids 2014;62:209-27. doi:10.1016/j.jmps.2013.09.021. Sixtieth anniversary issue in honor of Professor Rodney Hill

[3] Huang W, Yuan F-G, Jiang X. Flexoelectric effect, materials, and structures. In: Yuan F-G, editor. Structural health monitoring (SHM) in aerospace structures. Woodhead Publishing; 2016. p. 119-48.

[4] Ghasemi H, Park HS, Rabczuk T. A multi-material level set-based topology optimization of flexoelectric composites. Comput Methods Appl Mech Eng 2018;332:47-62. doi:10.1016/j.cma.2017.12.005.

[5] Lee HJ, Zhang S, Bar-Cohen Y, Sherrit S. High temperature, high power piezoelectric composite transducers. Sensors 2014;14(8):14526-52. doi:10.3390/s140814526.

[6] Stuber VL, Deutz DB, Bennett J, Cannel D, de Leeuw DM, van-der Zwaag S, et al. Flexible lead-free piezoelectric composite materials for energy harvesting applications. Energy Technol 2019;7(1):177-85. doi:10.1002/ente.201800419.

[7] Le Quang $\mathrm{H}$, He QC. The number and types of all possible rotational symmetries for flexoelectric tensors. Proc R Soc A 2011;467(2132):2369-86. doi:10.1098/rspa.2010.0521.

[8] Majdoub MS, Sharma P, Cagin T. Enhanced size-dependent piezoelectricity and elasticity in nanostructures due to the flexoelectric effect. Phys Rev B 2008;77:125424. doi:10.1103/PhysRevB.77.125424.

[9] Krichen S, Sharma P. Flexoelectricity: a perspective on an unusual electromechanical coupling. J Appl Mech 2016;83(3):030801-030801-5. doi:10.1115/1.4032378.

[10] Vasquez-Sancho F, Abdollahi A, Damjanovic D, Catalan G. Flexoelectricity in bones. Adv Mater 2018;30(9):1705316. doi:10.1002/adma.201705316.

[11] Lin Z-H, Yang Y, Wu JM, Liu Y, Zhang F, Wang ZL. Batio3 nanotubes-based flexible and transparent nanogenerators. J Phys Chem Lett 2012;3(23):3599-604. doi:10.1021/jz301805f.

[12] Shu L, Liang R, Rao Z, Fei L, Ke S, Wang Y. Flexoelectric materials and their related applications: a focused review. J Adv Ceram 2019;8(2):153-73. doi:10.1007/s40145-018-0311-3.

[13] Nguyen BH, Zhuang X, Rabczuk T. Numerical model for the characterization of maxwell-wagner relaxation in piezoelectric and flexoelectric composite material. Comput Struct 2018;208:75-91. doi:10.1016/j.compstruc.2018.05.006.
[14] Otero JA, Castillero JB, Ramos RR. Homogenization of heterogeneous piezoelectric medium. Mech Res Commun 1997;24(1):75-84. doi:10.1016/S0093-6413(96)00081-X.

[15] Guinovart-Díaz R, Rodríguez-Ramos R, Bravo-Castillero J, Federico JS, Otero J, Maugin G. A recursive asymptotic homogenization scheme for multi-phase fibrous elastic composites. Mech Mater 2005;37:1119-31. doi:10.1016/j.mechmat.2005.02.003.

[16] Castillero JB, Otero JA, Ramos RR, Bourgeat A. Asymptotic homogenization of laminated piezocomposite materials. Int J Solids Struct 1998;35(5):527-41. doi:10.1016/S0020-7683(97)00028-0.

[17] Tsalis D, Chatzigeorgiou G, Charalambakis N. Homogenization of structures with generalized periodicity. Composites Part B 2012(43 (2012)):2495-512.

[18] Guinovart-Sanjuán D, Vajravelu K, Rodríguez-Ramos R, Guinovart-Díaz R, Bravo-Castillero J, Lebon F, et al. Analysis of effective elastic properties for shell with complex geometrical shapes. Compos Struct 2018;203:278-85. doi:10.1016/j.compstruct.2018.07.036.

[19] Guinovart-Sanjuán D, Rodríguez-Ramos R, Guinovart-Díaz R, Bravo-Castillero J, Sabina FJ, Merodio J, Lebon F, Dumont S, Conci A. Effective properties of regular elastic laminated shell composite. Composite Part B 2016;87:12-20. doi:10.1016/j.compositesb.2015.09.051.

[20] Cioranescu D. An Introduction to Homogenization (Oxford Lecture Series in Mathematics and Its Applications). Oxford University Press; 2000. ISBN 0198565542. https://www.xarg.org/ref/a/0198565542/

[21] Bakhvalov N, Panasenko GP. Homogenisation: averaging processes in periodic media. Kluwer; 1989.

[22] Grekov AA, Kramarov SO, Kuprienko AA. Anomalous behavior of the two-phase lamellar piezoelectric texture. Ferroelectrics 1987;76(1):43-8. doi:10.1080/00150198708009022.

[23] Guinovart-Sanjuán D, Rodríguez-Ramos R, Guinovart-Díaz R, BravoCastillero J, Sabina FJ, Merodio J, et al. Effective properties of regular elastic laminated shell composite. Composites Part B 2016;87:12-20. doi:10.1016/j.compositesb.2015.09.051.

[24] Guinovart-Sanjuán D, Merodio J, López-Realpozo JC, Vajravelu K, RodríguezRamos R, Guinovart-Díaz R, et al. Asymptotic homogenization applied to flexoelectric rods. Materials 2019;12(2). doi:10.3390/ma12020232.

[25] Bravo-Castillero J, Rodríguez-Ramos R, Mechkour H, Otero JA, Sabina FJ. Homogenization of magneto-electro-elastic multilaminated materials. Q J Mech ApplMath 2008;61(3):311-32. doi:10.1093/qjmam/hbn010.

[26] Royer D, Dieulesaint E. Elastic waves in solids i. Berlin Heidelberg: Springer; 2000.

[27] Maranganti R, Sharma P. Atomistic determination of flexoelectric properties of crystalline dielectrics. Phys Rev B 2009;80:054109. doi:10.1103/PhysRevB.80.054109.

[28] Shu L, Wei X, Pang T, Yao X, Wang C. Symmetry of flexoelectric coefficients in crystalline medium. J Appl Phys 2011;110(10):104106. doi:10.1063/1.3662196.

[29] Tsalis D, Chatzigeorgiou G, Charalambakis N. Effective behavior of thermoelastic tubes with wavy layers. Composites Part B 2016;99:173-87. doi:10.1016/j.compositesb.2016.05.043.

[30] Wu, Sheng B-Y, Hao X-Q, Yang. Effective media properties of hyperuniform disordered composite materials. PLOS ONE 2017;12(10):1-13. doi:10.1371/journal.pone.0185921.

[31] Berger H, Kari S, Gabbert U, Rodríguez-Ramos R, Bravo-Castillero J, GuinovartDíaz R. A comprehensive numerical homogenisation technique for calculating effective coefficients of uniaxial piezoelectric fibre composites. Materials Science and Engineering: A 2005;412(1):53-60. doi:10.1016/j.msea.2005.08.035. International conference on recent advances in composite materials

[32] Wang B, Gu Y, Zhang S, Chen L-Q. Flexoelectricity in solids: progress, challenges, and perspectives. Prog Mater Sci 2019;106:100570. doi:10.1016/j.pmatsci.2019.05.003.

[33] Sixto-Camacho LM, Bravo-Castillero J, Brenner R, Guinovart-Díaz R, Mechkour H, Rodríguez-Ramos R, Sabina FJ. Asymptotic homogenization of periodic thermomagneto-electro-elastic heterogeneous media. Computers \& Mathematics with Applications 2013;66(10):2056-74. doi:10.1016/j.camwa.2013.08.027. ICNC-FSKD 2012 